\title{
A Dynamic Semiparametric Proportional Hazard Model
}

Hautsch, Nikolaus; Gerhard, Frank

Publication date:

2006

Document version

Publisher's PDF, also known as Version of record

Citation for published version (APA):

Hautsch, N., \& Gerhard, F. (2006). A Dynamic Semiparametric Proportional Hazard Model. Department of Economics, University of Copenhagen. 


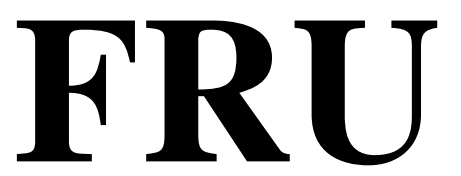

Finance Research Unit

\title{
A Dynamic Semiparametric Proportional Hazard Model
}

\author{
Frank Gerhard and Nikolaus Hautsch
}

No. 2006/05

Finance Research Unit Department of Economics University of Copenhagen http://www.econ.ku.dk/FRU 


\title{
A DYNAMIC SEMIPARAMETRIC PROPORTIONAL HAZARD MODEL
}

\author{
FRANK GERHARD
}

GLOBAL FINANCIAL RISK MANAGEMENT

BARCLAYS CAPITAL, LONDON

NIKOLAUS HAUTSCH*

DEPARTMENT OF ECONOMICS

UNIVERSITY OF COPENHAGEN

\begin{abstract}
This paper proposes a dynamic proportional hazard (PH) model with non-specified baseline hazard for the modelling of autoregressive duration processes. A categorization of the durations allows us to reformulate the $\mathrm{PH}$ model as an ordered response model based on extreme value distributed errors. In order to capture persistent serial dependence in the duration process, we extend the model by an observation driven ARMA dynamic based on generalized errors. We illustrate the maximum likelihood estimation of both the model parameters and discrete points of the underlying unspecified baseline survivor function. The dynamic properties of the model as well as an assessment of the estimation quality is investigated in a Monte Carlo study. It is illustrated that the model is a useful approach to estimate conditional failure probabilities based on (persistent) serial dependent duration data which might be subject to censoring structures. In an empirical study based on financial transaction data we present an application of the model to estimate conditional asset price change probabilities. Evaluating the forecasting properties of the model, it is shown that the proposed approach is a promising competitor to well-established ACD type models.
\end{abstract}

\section{INTRODUCTION}

Recent literature on high-frequency finance inspired growing interest in dynamic duration models. They serve as fundamental tools to capture the irregular spacing of financial market events, such as e.g. trade or quote arrivals, or the occurrence of cumulative price changes or cumulative trading volumes of a given size. Modelling the time between these events, i.e. the so-called financial durations, allows one to analyze market microstructure relations and to quantify the instantaneous volatility and liquidity as well as the dynamic interactions thereof. Since financial durations typically reveal persistent autoregressive structures, the specification of dynamic duration models requires to combine features from (nonlinear) time series approaches with those of classical duration models.

November 6, 2006.

JEL Classifications: C22 C25 C41 G14.

Keywords: autoregressive duration models, dynamic ordered response models, generalized residuals, censoring.

* Email: Nikolaus.Hautsch@econ.ku.dk. Address: Studiestraede 6, DK-1455 Copenhagen K, Tel.: +45 3532 30 22. Fax: +45353230 00 .

For valuable comments we would like to thank Luc Bauwens, Sir David Cox, Robert Engle, David Veredas, Joachim Inkmann, Bent Nielsen, Neil Shephard and Winfried Pohlmeier. Early versions of this paper have been presented at the 8th World Congress of the Econometric Society, Seattle, 2000 and the CoFE Conference on Intertemporal Finance, Konstanz, 2000. All remaining errors are our sole responsibility. 
In this paper, we introduce a new type of dynamic duration model which has its origin in duration analysis. The main contribution is to propose a dynamic extension of the Cox (1972) semiparametric proportional hazard $(\mathrm{PH})$ model, where the baseline hazard function is nonspecified. The resulting model is called Semiparametric Autoregressive Conditional Proportional Hazard (SACPH) model and is shown to be a useful approach to model data which reveal characteristics of classical duration data, like censoring structures or unobservable heterogeneity effects, but also persistent autoregressive structures.

A seminal contribution to the literature on dynamic duration models was the autoregressive conditional duration (ACD) model introduced by Engle and Russell (1998) and Engle (2000) which originates from the time series literature on GARCH and ARMA processes and was the starting point for a wide range of extensions. In the basis ACD specification, the durations standardized by their conditional mean are assumed to be i.i.d., where the conditional duration mean is assumed to follow a linear autoregressive process. One string of extensions focusses on generalizations of the specification of the conditional mean function leading to logarithmic specifications (Bauwens and Giot, 2000), (augmented) Box-Cox type specifications (Dufour and Engle, 2000, Fernandes and Grammig, 2006, Hautsch, 2003, 2006) as well as regime switching specifications (Zhang, Russell, and Tsay, 2001, Hujer and Vuletic, 2005, Meitz and Teräsvirta, 2006). A further string of the literature focusses on generalizations of the distribution properties. Whereas Engle and Russell (1998) assume that the standardized durations are exponentially or Weibull distributed, Grammig and Maurer (2000), Lunde (2000) and Hautsch (2003) propose Burr, generalized gamma and generalized F distributions, respectively. Further approaches deal with the presence of long range dependence (Jasiak, 1998), the modelling of a stochastic conditional duration mean process (Bauwens and Veredas, 2004) and the nonparametric treatment of seasonality effects (Veredas, Rodriguez-Poo, and Espasa, 2002). All these specifications belong to the class of accelerated failure time (AFT) models $^{1}$, where observable characteristics, such as lagged durations or functions thereof, serve as accelerators (or decelerators) of the time scale on which the durations are measured ${ }^{2}$ and have their origin in (nonlinear) ARMA-GARCH type time series models.

However, so far it is not well understood which elements of time series models and classical duration models should be optimally combined in the specification of a dynamic duration model and from which class of approaches to start with. Actually, Bauwens et al. (2004) illustrate that in many applications even quite flexibly parameterized (AFT type) dynamic duration models fail to completely capture the distribution properties of (financial) durations. A further drawback of the ACD model is that it does not allow to account for censoring structures in duration processes. Censoring is apparent whenever the beginning or the end of a duration, and thus the

\footnotetext{
${ }^{1}$ See e.g. Kalbfleisch and Prentice (1980).

${ }^{2}$ See Engle and Russell (1998) or Hautsch (2004) for an illustration of this relation.
} 
exact length of the spell cannot be completely observed and can only be approximated by upper and/or lower boundaries. E.g., in the context of financial duration processes, censoring can occur because of non-trading periods such as nights, weekends, holidays or trading halts. Furthermore, particularly whenever one is not only interested in predictions of the conditional duration mean but also in estimates of conditional failure probabilities, i.e. the conditional probability to observe the next event arrival in a certain time interval, parametric specifications of the (conditional) density function, as required in the ACD framework, might be too restrictive.

Motivated by these observations we propose a model which originates from the duration literature. An obvious starting point is the Cox (1972) PH model which is still the workhorse in classical survival analysis. ${ }^{3}$ The main contribution of this paper is to extend the Cox model to allow for persistent serial dependence in the duration process. In particular, we assume that the hazard function is specified as a multiplicative function of a so-called baseline hazard component and a (parametric) function of observable characteristics (including covariates and model dynamics). The baseline hazard captures the shape of the hazard function during the duration spell as a deterministic function of time. A continuous-time counterpart to this type of model has been proposed by Russell (1999) who models the hazard (or intensity) itself in terms of an ACD type autoregressive process. However, while Russell (1999) specifies the baseline intensity parametrically, we leave it non-specified and suggest to estimate it semiparametrically. In the paper, we illustrate that this flexibility is required to capture the distribution properties of (dynamic) duration data.

We use the well-known result that any $\mathrm{PH}$ model can be re-formulated as a linear regression model with extreme value distributed error terms and the observable characteristics serving as regressors. ${ }^{4}$ By employing a categorization scheme for the underlying durations we exploit the relationship between ordered response models and models for grouped duration data (see e.g. Sueyoshi, 1995). In this context, the PH model can be interpreted as an ordered response model based on an extreme value distribution, where the left-hand variable in the latent equation corresponds to the log of the integral of the baseline hazard function computed over the time interval from the previous until the most recent event arrival (the so-called log integrated baseline hazard). The major idea of the SACPH model is to extend this (latent) regression model by allowing for ARMA type dynamics in terms of the log integrated baseline hazard. In order to overcome the problem that the latter is not directly observable and to avoid the computation of cumbersome high-dimensional integrals, we assume the model dynamics to be observation driven, i.e. to be computable based on the observable history of the process. In this context, we rely on the concept of generalized errors in the spirit of Gouriéroux et al. (1987) and replace the true (unobservable) errors by their conditional expectations.

\footnotetext{
${ }^{3}$ See e.g. Kalbfleisch and Prentice (1980), Kiefer (1988) of Lancaster (1997).

${ }^{4}$ See e.g. Kiefer (1988).
} 
An important advantage of the proposed model is that it allows for straightforward maximum likelihood estimation of the model parameters as well as discrete points of the underlying (non-specified) baseline survivor function. Since analytical expressions for the theoretical autocorrelation function implied by the SACPH model are not available, we compute them numerically for different parameter settings. The effects of the chosen categorization on the estimation quality is investigated on the basis of a Monte Carlo study. Results indicate that the estimation performs nicely for a reasonable number of categories and a rather moderate sample size.

The SACPH model is a flexible approach to model various types of dynamic point processes. Since the model dynamics are based on generalized errors, the SACPH model provides a convenient framework to account for censored observations which enter the model dynamics in terms of conditional expectations. This is particularly important when e.g. price intensities, i.e. the times until the occurrence of a cumulative absolute price change of a given size, are analyzed. Price intensities provide a powerful way to derive volatility measures which do not require a (fixed interval) time aggregation scheme. ${ }^{5}$ However, since prices are unobservable during non-trading periods, the exact length of the resulting duration spells are not necessarily observed. In the paper, we illustrate an application of the SACPH model to this kind of problem by estimating (conditional) price change probabilities in the trading process of the German BUND future traded at the EUREX. We focus on cumulative absolute price movements which last on average over several trading days and thus reflect distinct censoring structures due to non-trading periods. It turns out that the model does a good job in capturing the persistence in the data and provides estimates of conditional failure probabilities which are quite robust with respect to the choice of the underlying categorization scheme of the data. In an evaluation study, we compare the in-sample and out-of-sample forecasting properties of the SACPH model with those of various ACD models. In the given application, it is shown that the SACPH model provides a significantly better in-sample goodness-of-fit while simultaneously yielding out-of-sample forecasts which are slightly worse but still qualitatively similar to those of ACD models.

Further potential applications of the model are the quantification of the conditional probability that a limit order is executed within a certain time span given the state of the market and the length of previous (possibly censored) limit order spells. Alternative applications might be quantifications of the intensity of bond rating changes (see e.g. Koopman, Lucas, and Monteiro, 2005) or the probability for central bank interventions. In industrial economics, an interesting application might be the quantification of the intensity of patent activity as a measure for the inventive activity of a firm (see e.g. Blazsek and Escribano, 2005).

The outline of the remainder of the paper is as follows: In Section 2, we briefly review the original PH model and the relationship between grouped PH models and ordered response models. Section 3 illustrates the extension from the standard PH model to the SACPH model.

\footnotetext{
${ }^{5}$ See Engle and Russell (1998) or Gerhard and Hautsch (2002).
} 
Theoretical properties are discussed in Section 4. Here, we analyze the autocorrelation structure implied by the model, as well as small sample effects and effects of the underlying categorization on the estimation quality. Section 5 extends the SACPH model by accounting for unobserved heterogeneity and censoring structures in the data. Diagnostic tests for the model are given in Section 6. In Section 7, an application of the SACPH approach based on price durations as well as a forecasting comparison with the ACD model are presented. Finally, Section 8 concludes.

\section{The Proportional Hazard Model}

Let $\left\{t_{i}\right\}_{i \in\{1,2, \ldots, n\}}$ be a sequence of random event arrival times with $t_{i}<t_{i+1}$. Furthermore, define $x_{i}:=t_{i}-t_{i-1}$ as the realization of a random variable $X_{i}$ representing the duration between two consecutive events. Following the duration literature ${ }^{6}$ the (conditional) hazard function is defined as

$$
\lambda\left(x ; \mathcal{F}_{i-1}\right)=\lim _{\Delta \downarrow 0} \frac{1}{\Delta} \operatorname{Pr}\left[x \leq x_{i}<x+\Delta \mid x_{i} \geq x ; \mathcal{F}_{i-1}\right],
$$

where $\mathcal{F}_{i}$ denotes the information set up to (inclusive) $t_{i}$. The hazard function can be heuristically interpreted as the conditional instantaneous arrival rate given the information set and the time elapsed since the beginning of the duration spell.

The standard PH model has its origin in cross-sectional survival analysis in biostatistics ${ }^{7}$ and labor economics ${ }^{8}$ and is specified as the product of a baseline hazard function $\lambda_{0}(x)>0$ and a strictly positive function of a vector of covariates $z$ with coefficients $\gamma$. It is given by

$$
\lambda\left(x ; z_{i-1}\right)=\lambda_{0}(x) \exp \left(-z_{i-1}^{\prime} \gamma\right)
$$

where the covariates are assumed to be observable at the beginning of the duration spell. If the baseline hazard function is fully parameterized, e.g. based on a Weibull distribution,

$$
\lambda_{0}(x)=p x^{p-1},
$$

where $p>0$ determines the shape parameter, the model is straightforwardly estimated by maximum likelihood (ML). In classical duration literature, it is well known that in a full information ML approach, a consistent estimation of $\gamma$ requires the correct specification of $\lambda_{0} \cdot{ }^{9}$ However, often parametric distributions are not sufficient to correctly capture the hazard shape. For that reason, Cox $(1972,1975)$ suggested to remain the baseline hazard $\lambda_{0}$ completely unspecified and proposed a partial likelihood approach in order to consistently estimate $\gamma$ without the knowledge

\footnotetext{
${ }^{6}$ See e.g. Kalbfleisch and Prentice (1980), Cox and Isham (1980), Kiefer (1988) or Lancaster (1997).

${ }^{7}$ See, for example, Kalbfleisch and Prentice (1980), Cox and Oakes (1984), or the survey by Oakes (2001).

${ }^{8} \mathrm{~A}$ well known example is the analysis of the length of unemployment spells which is studied by a wide range of theoretical and empirical papers, see e.g. Lancaster (1979), Nickell (1979), Heckmann and Singer (1984), Moffitt (1985), Honoré (1990), Meyer (1990), Han and Hausman (1990), Gritz (1993), McCall (1996) or van den Berg and van der Klaauw (2001) among many others.

${ }^{9}$ See e.g. Heckmann and Singer (1984).
} 
of the baseline hazard $\lambda_{0}$. The estimation of the baseline hazard itself follows from a modification of the nonparametric estimator by Kaplan and Meier (1958) proposed by Breslow (1972, 1974).

An alternative way to estimate the $\mathrm{PH}$ model relies on the close relationship between quantal response models and PH models for grouped durations models (see Han and Hausman, 1990, Meyer, 1990, or Sueyoshi, 1995). By exploiting the relation between the (conditional) survivor function $S\left(x_{i} \mid \mathcal{F}_{i-1}\right)$ and the hazard function, $S\left(x_{i} \mid \mathcal{F}_{i-1}\right)=\operatorname{Pr}\left(X_{i} \geq x_{i} \mid \mathcal{F}_{i-1}\right)=$ $\exp \left[-\int_{0}^{x_{i}} \lambda\left(s ; \mathcal{F}_{i-1}\right) d s\right]$, it is straightforwardly shown that

$$
\Lambda\left(x_{i}\right):=\int_{0}^{x_{i}} \lambda\left(s ; \mathcal{F}_{i-1}\right) d s \sim \text { i.i.d. } \operatorname{Exp}(1),
$$

where $\Lambda\left(x_{i}\right)$ denotes the integrated hazard function. ${ }^{10}$ Then, the PH model can be rewritten as a regression model in terms of the log integrated baseline hazard,

$$
\ln \Lambda_{0}\left(x_{i}\right):=\ln \int_{0}^{x_{i}} \lambda_{0}\left(s ; \mathcal{F}_{i-1}\right) d s=z_{i-1}^{\prime} \gamma+\epsilon_{i}^{*}, \quad i=1, \ldots, n,
$$

where $\epsilon_{i}^{*}:=\ln \Lambda\left(x_{i}\right)$ follows an i.i.d. standard extreme value type I distribution (standard Gumbel (minimum) distribution) with mean $\mathrm{E}\left[\epsilon_{i}^{*}\right]=-0.5772$, variance $\operatorname{Var}\left[\epsilon_{i}^{*}\right]=\pi^{2} / 6$ as well as probability density function (p.d.f.) and cumulative distribution function (c.d.f) given by

$$
\begin{aligned}
& f_{\epsilon^{*}}(x)=\exp (x-\exp (x)), \\
& F_{\epsilon^{*}}(x)=\exp (-\exp (x)) .
\end{aligned}
$$

This formulation allows us to interpret the $\mathrm{PH}$ model as a linear regression model with extreme value distributed error terms and the log integrated baseline hazard serving as left hand variable. In the special case where $\lambda_{0}(\cdot)$ is fully parameterized, and thus $\ln \Lambda_{0}\left(x_{i}\right)$ can be directly computed as a function of $x_{i}$, the PH model can be consistently estimated even by OLS (see Kiefer, 1988 for a nice illustration.) In contrast, if $\lambda_{0}(\cdot)$ is left unspecified, the transformation from $x_{i}$ to $\ln \Lambda_{0}\left(x_{i}\right)$ is unknown and thus $\ln \Lambda_{0}\left(x_{i}\right)$ has to be treated as a latent variable.

The major idea of Han and Hausman (1990) is to group the durations according to an exogenously given categorization scheme and to interpret the $\mathrm{PH}$ model as an ordered response model based on extreme value distributed errors with (5) serving as the latent variable equation. Following this approach, we partition the observed durations $x_{i}$ into $K$ categories, where $\bar{x}_{k}, k=$ $1, \ldots, K-1$ denote the chosen category bounds and define

$$
\mu_{k}^{*}:=\ln \Lambda_{0}\left(\bar{x}_{k}\right), \quad k=1, \ldots, K-1,
$$

as the value of the latent variable $\ln \Lambda_{0}(\cdot)$ at the realization of the (observable) category bound $\bar{x}_{k}$. Furthermore, define

$$
x_{i}^{d}:=k \cdot \mathbb{1}_{\left\{\bar{x}_{k-1}<x_{i} \leq \bar{x}_{k}\right\}}, \quad k=1, \ldots, K,
$$

\footnotetext{
${ }^{10}$ See e.g. Kiefer (1988). Note that this relation holds straightforwardly as long as we exclude the case of time-varying covariates and a continuous updating of the information set. The latter case is not easily taken into account in the given semiparametric framework and is left for future research.
} 
with $\bar{x}_{0}=0$ and $\bar{x}_{K}=\infty$ as an ordered integer variable indicating the observed category. Then, the (conditional) probability for observing a duration lying in category $k$ is computed as

$$
\operatorname{Pr}\left[x_{i}^{d}=k \mid z_{i-1}\right]= \begin{cases}F_{\epsilon^{*}}\left(\mu_{1}^{*}-z_{i-1}^{\prime} \gamma\right) & \text { if } x_{i}^{d}=1, \\ F_{\epsilon^{*}}\left(\mu_{2}^{*}-z_{i-1}^{\prime} \gamma\right)-F_{\epsilon^{*}}\left(\mu_{1}^{*}-z_{i-1}^{\prime} \gamma\right) & \text { if } x_{i}^{d}=2, \\ \vdots & \text { if } x_{i}^{d}=K . \\ 1-F_{\epsilon^{*}}\left(\mu_{K-1}^{*}-z_{i-1}^{\prime} \gamma\right) & \text {. }\end{cases}
$$

The direct relationship between the latent thresholds $\mu_{k}^{*}$ and the log integrated baseline hazard $\ln \Lambda_{0}(\cdot)$ is one of the main advantages of this approach.By employing the relation between (baseline) survivor function and integrated (baseline) hazard function, the unknown baseline survivor function $S_{0}$ can be estimated at the $K-1$ discrete points by

$$
S_{0}\left(\bar{x}_{k}\right)=\exp \left[-\int_{0}^{\bar{x}_{k}} \lambda_{0}(s) d s\right]=\exp \left[-\Lambda_{0}\left(\bar{x}_{k}\right)\right]=\exp \left(-\exp \left(\mu_{k}^{*}\right)\right), \quad k=1, \ldots, K-1 .
$$

Naturally, a categorization approach induces some loss of information. However, the important result of Cox (1975) that $\gamma$ can be estimated consistently without a specification of $\lambda_{0}$ also holds in this categorization framework. In particular Meyer (1990) illustrates that the consistency of $\hat{\gamma}$ does not depend on the chosen categorization scheme and the number of the underlying categories. ${ }^{11}$

The resulting log likelihood function has the well known form of an ordered response model based on the standard extreme value distribution

$$
\ln \mathcal{L}(W ; \theta)=\sum_{i=1}^{n} \sum_{k=1}^{K} \mathbb{1}_{\left\{x_{i}^{d}=k\right\}} \ln \operatorname{Pr}\left[x_{i}^{d}=k \mid z_{i-1}\right],
$$

where $W$ denotes the data matrix and $\theta$ the parameter vector.

The idea of interpreting (5) as the latent equation of an ordered response model will be the starting point for a dynamic extension. This will be illustrated in the following section.

\section{The Semiparametric Autoregressive Conditional Proportional Hazard Model}

In order to illustrate a dynamic extension of the semiparametric PH model, we reformulate eq. (5) in more general form as

$$
\ln \Lambda_{0, i}=\phi_{i}+\epsilon_{i}^{*}
$$

where $\Lambda_{0, i}:=\Lambda_{0}\left(x_{i}\right)$ and $\phi_{i}$ denotes a function capturing the dynamics of the process as well as potential covariates. Recall that $\Lambda_{0, i}$ is a function of the duration $x_{i}$ in dependence of the unknown form of $\lambda_{0}$. Hence, (13) can be interpreted as a latent variable model.

The appropriate parameterization of $\phi_{i}$ in a dynamic context is not obvious. Our objective is to let $\phi_{i}$ fulfill two major tasks: (i) $\phi_{i}$ has to capture persistent serial dependence in the duration process, and, (ii) the parameterization of $\phi_{i}$ should lead to a computationally tractable

\footnotetext{
${ }^{11}$ Nevertheless, the efficiency of the estimator is affected by the chosen categorization.
} 
model. Consider as an illustration a parameterization of $\phi_{i}$ as given by

$$
\begin{aligned}
\phi_{i} & =\psi_{i}+z_{i-1}^{\prime} \gamma \\
\psi_{i} & =\sum_{j=1}^{p} \tilde{\alpha}_{j}\left(\psi_{i-j}+\epsilon_{i-j}^{*}\right)+\sum_{j=1}^{q} \tilde{\beta}_{j} \epsilon_{i-j}^{*} \\
& =\sum_{j=1}^{p} \tilde{\alpha}_{j}\left(\ln \Lambda_{0, i-j}-z_{i-j-1}^{\prime} \gamma+\sum_{j=1}^{q} \tilde{\beta}_{j} \epsilon_{i-j}^{*} .\right.
\end{aligned}
$$

This specification corresponds to an $\operatorname{ARMA}(p, q)$ model in terms of the log integrated baseline hazard, $\ln \Lambda_{0, i}$, which is augmented by covariates. However, the major difficulty in this context is that $\Lambda_{0, i}$ is unobservable and thus the dynamics are attached to a latent variable. In this context maximum likelihood estimation requires to integrate out the latent variable leading to an integral with the dimension equal to the sample size. Even though efficient numerical methods have been developed and successfully applied in recent literature ${ }^{12}$ they are still computationally cumbersome for very long time series.

Therefore, we propose an alternative parameterization of $\phi_{i}$ by specifying an observation driven dynamic which overcomes the problem that $\Lambda_{0, i}$ is not directly observable and prevents us from computing cumbersome high-dimensional integrals. Our approach is based on the categorization illustrated in the previous section and exploits the fact that, according to (8), the estimated thresholds $\mu_{k}^{*}, k=1, \ldots, K$, correspond per construction to estimates of $\ln \Lambda_{0, i}$ at the realizations of the corresponding category bounds $\bar{x}_{k}$. The major idea is to replace the unobservable (extreme value distributed) error terms $\epsilon_{i}^{*}$ by their conditional expectation

$$
e_{i}:=\mathrm{E}\left[\epsilon_{i}^{*} \mid \mathcal{F}_{i}^{d}\right]
$$

where

$$
\mathcal{F}_{i}^{d}:=\sigma\left(x_{i}^{d}, x_{i-1}^{d}, \ldots, x_{1}^{d}, z_{i}, z_{i-1}, \ldots, z_{1}\right)
$$

denotes the filtration generated by the categorized durations and possible covariates. Thus, $e_{i}$ can be interpreted as a generalized error in the sense of Gouriéroux et al (1987).

Then, instead of specifying $\psi_{i}$ in terms of the original (unobservable) errors $\epsilon_{i}^{*}$, we propose to specify it in terms of an observation driven ARMA type recursion given by

$$
\psi_{i}=\sum_{j=1}^{p} \alpha_{j}\left(\psi_{i-j}+e_{i-j}\right)+\sum_{j=1}^{q} \beta_{j} e_{i-j},
$$

which is conditioned on an initial value $\psi_{0}$. Since it is built on an ARMA structure based on the conditional expectations of the latent errors given the observable categorized durations, it allows us to compute $\psi_{i}$ recursively using a prediction error decomposition and thus prevents us from computing cumbersome high-dimensional integrals. We call the model as characterized by eq. (13), (14) and (17) Semiparametric Autoregressive Conditional Proportional Hazard (SACPH) model. The stationarity conditions for the SACPH model correspond to the well

\footnotetext{
${ }^{12}$ See e.g. Richard (1998), Liesenfeld and Richard (2003) or Bauwens and Hautsch (2006).
} 
known stationarity conditions of a standard ARMA model. In terms of the hazard function the SACPH model is written as

$$
\lambda\left(x ; \mathcal{F}_{i-1}^{d}\right)=\lambda_{0}(x) \exp \left(-\phi_{i}\right) .
$$

In order to illustrate the principle of the model, we consider the special case of a parametric ACPH model based on a fully parameterized $\lambda_{0}$. In this case, $\lambda_{0}$ and thus the transformation from $x_{i}$ to $\Lambda_{0, i}$ are known. Then, $e_{i}=\epsilon_{i}^{*}$ and eq. (17) corresponds to an ARMA process in terms of the $\log$ integrated hazard as shown in eq. (15) with parameters $\alpha_{j}=\tilde{\alpha}_{j}$ and $\beta_{j}=\tilde{\beta}_{j}$. Consider for illustration a Weibull specification for the baseline hazard, eq. (3). Then, $\ln \Lambda_{0, i}=p \ln x_{i}$ and the Weibull $\operatorname{ACPH}(p, q)$ model can be written as an $\operatorname{ARMA}(p, q)$ model for log durations based on a standard extreme value distribution, i.e.

$$
\begin{aligned}
\ln x_{i} & =\phi_{i}+\epsilon_{i}^{*} \\
\phi_{i} & =\sum_{j=1}^{p} \alpha_{j}\left(\ln x_{i-j}-z_{i-j-1}^{\prime} \frac{\gamma}{p}\right)+\sum_{j=1}^{q} \frac{\beta_{j}}{p} \epsilon_{i-1}^{*}+z_{i-1}^{\prime} \frac{\gamma}{p}+\frac{\epsilon_{i}^{*}}{p} .
\end{aligned}
$$

An important advantage of the proposed dynamic is that it allows for a straightforward computation of the likelihood function without requiring the use of simulation methods. Thus, a simple maximum likelihood estimator of the model parameters as well as of the thresholds $\mu_{k}^{*}$ is directly available.

The computation of the log likelihood function requires to calculate the generalized errors $e_{i}$ by

$$
\begin{aligned}
e_{i} & :=\mathrm{E}\left[\epsilon_{i}^{*} \mid \mathcal{F}_{i-1}^{d}\right]=\mathrm{E}\left[\epsilon_{i}^{*} \mid x_{i}^{d}, \phi_{i}\right] \\
& = \begin{cases}\frac{\kappa\left(-\infty, \nu_{i, 1}\right)}{F_{\epsilon^{*}\left(\nu_{i, 1}\right)}} & \text { if } x_{i}^{d}=1, \\
F_{\epsilon^{*}\left(\nu_{i, k-1}, \nu_{i, k}\right)-F_{\epsilon^{*}}\left(\nu_{i, k-1}\right)} & \text { if } x_{i}^{d} \in\{2, \ldots, K-1\}, \\
\frac{\kappa\left(\nu_{i, K-1}, \infty\right)}{1-F_{\epsilon^{*}\left(\nu_{i, K-1}\right)}} & \text { if } x_{i}^{d}=K,\end{cases}
\end{aligned}
$$

where $\nu_{i, k}:=\mu_{k}^{*}-\phi_{i}$ and $\kappa\left(s_{1}, s_{2}\right):=\int_{s_{1}}^{s_{2}} u f_{\epsilon^{*}}(u) d u \cdot{ }^{13}$

Since the observation driven dynamic enables us to use the standard prediction error decomposition, the likelihood is evaluated in an iterative fashion: The function $\psi_{i}$ is initialized with its unconditional expectation $\psi_{0}:=\mathrm{E}\left[\psi_{i}\right]$. Then, based on the recursion, eq. (17), as well as the definition of the generalized errors, eq. (21), the likelihood contribution of observation $i$ given the observation rule eq. (9) is computed as follows

$$
\operatorname{Pr}\left[x_{i}^{d}=k \mid \mathcal{F}_{i-1}^{d}\right]= \begin{cases}F_{\epsilon^{*}}\left(\mu_{1}^{*}-\phi_{i}\right) & \text { if } x_{i}^{d}=1, \\ F_{\epsilon^{*}}\left(\mu_{2}^{*}-\phi_{i}\right)-F_{\epsilon^{*}}\left(\mu_{1}^{*}-\phi_{i}\right) & \text { if } x_{i}^{d}=2, \\ \vdots & \text { if } x_{i}^{d}=K . \\ 1-F_{\epsilon^{*}}\left(\mu_{K-1}^{*}-\phi_{i}\right) & \text {. }\end{cases}
$$

Under the standard regularity conditions for maximum likelihood estimation and the assumption that the data generating process (DGP) is given by (13), (14) and (17), the model parameters

\footnotetext{
${ }^{13}$ For an extended discussion of generalized errors, see Gouriéroux et al. (1987).
} 
are consistently estimated. However, a requirement for consistency is that the true DGP is based on generalized errors as computed in eq. (21). Naturally, this might be a restrictive assumption, particularly when the chosen categorization is relatively rough.

In order to illustrate the model properties in more detail, assume that the true DGP corresponds to (15), in which $\ln \Lambda_{0, i}$ follows an ARMA process based on the true errors $\epsilon_{i}^{*}$ and the parameters $\tilde{\alpha}_{j}$ and $\tilde{\beta}_{j}$. In this case, the SACPH model as given by (14) and (17) does generally not provide consistent estimates of $\tilde{\alpha}_{j}$ and $\tilde{\beta}_{j}$. However, the higher the number of categories, the closer is the difference between the generalized error $e_{i}$ and its true (unobservable) counterpart $\epsilon_{i}^{*}$. If $K \rightarrow \infty, S_{0}(x)$ is estimated at an infinite number of points, and thus is quasi-observable. Then, the SACPH model based on generalized errors, eq. (17) converges to its counterpart based on true errors, eq. (15), with parameters $\alpha_{j}=\tilde{\alpha}_{j}$ and $\beta_{j}=\tilde{\beta}_{j}$. Therefore, (15) can be seen as the limiting case in which the log integrated baseline hazard $\ln \Lambda_{0, i}$ is completely observable and follows an ARMA dynamic based on extreme value distributed errors. Hence, as long as if we assume (15) to be the true DGP, $\hat{\alpha}_{j}$ and $\hat{\beta}_{j}$ are generally not consistent estimates for $\tilde{\alpha}_{j}$ and $\tilde{\beta}_{j}$, but can be only seen as approximations. The quality of this approximation is explored in the next section on the basis of a Monte Carlo study. It will be illustrated that for a moderate number of categories and a reasonable sample size, the estimates $\hat{\alpha}_{j}$ and $\hat{\beta}_{j}$ are nearly unbiased for $\tilde{\alpha}_{j}$ and $\tilde{\beta}_{j}$. Hence, it turns out that the SACPH process provides a sufficiently good approximation of an ARMA process in terms of the log integrated baseline hazard, $\ln \Lambda_{0, i}$, based on extreme value distributed errors.

\section{Properties of the SACPH Model}

4.1. Autocorrelation Structure. Since the dynamics of the model are based on a latent structure, an important question is how the autoregressive parameters can be interpreted with respect to the observable duration process. This issue will be considered in more details in the present subsection.

The main difficulty is that no closed form expression for the generalized errors and the p.d.f of the latent variable $\Lambda_{0, i}$ can be given, so that one needs to resort to numerical methods to evaluate the model's autocorrelation function (ACF). For this reason, we conduct a simulation study, where we generate three different processes. Firstly, using predetermined autoregressive parameters $\alpha$ and $\beta^{14}$ and random draws from a standard extreme value distribution, we simulate an $\operatorname{SACPH}(1,1)$ process according to (13), (14) and (17). Since the unconditional quantiles of the resulting distribution of $\ln \Lambda_{0, i}$ endogenously depend on the chosen categorization, we employ an iteration procedure in order to find category bounds which exactly resemble given unconditional quantiles of $\ln \Lambda_{0, i}$. Then, the SACPH process is simulated using three different categorizations.

\footnotetext{
${ }^{14}$ For simplicity, we assume that $\gamma=0$.
} 
The first grouping is based on only two categories, in particular, below and above the 0.5quantile of $\ln \Lambda_{0, i}$. Correspondingly, the other categorizations are based on the 0.25-, 0.5- and 0.75 -quantiles, as well as on the $0.1-, 0.2-, \ldots, 0.9$-quantiles. The former categorization can be seen as the worst possible approximation of the true baseline hazard $\lambda_{0}$ one could possible think of in the context of the given model and the latter being a more realistic case of using a moderate number of thresholds. Secondly, we compute the resulting (observable) duration process implied by the simulated realizations of $\ln \Lambda_{0, i}$. In this context, we have to impose a parametric assumption for the baseline hazard $\lambda_{0}$. In order to keep the analysis simple, we assume a Weibull hazard with parameter $p=0.5 .{ }^{15}$ Thirdly, as benchmark case we also simulate process (15) for $\alpha=\tilde{\alpha}$ and $\beta=\tilde{\beta}$. As discussed in the previous section, this process corresponds to the limiting case of an SACPH process when $K \rightarrow \infty$, i.e., when $\lambda_{0}$ is quasi-observable.
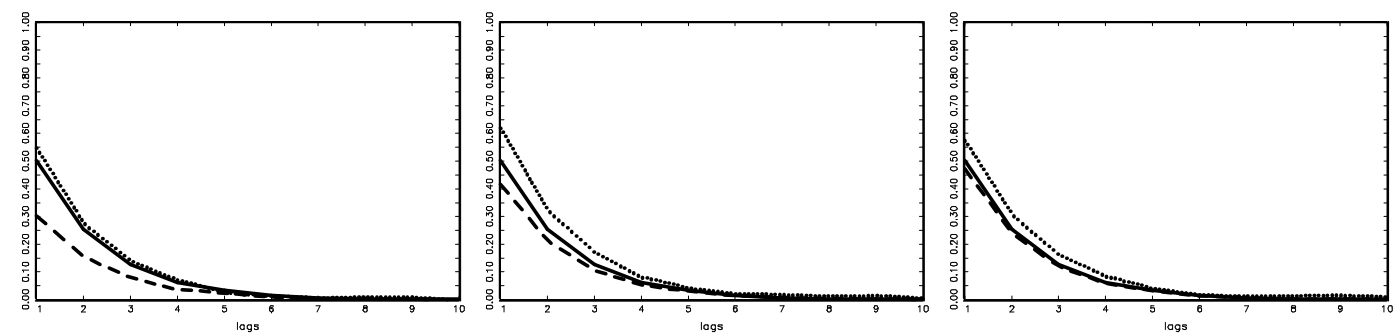

Figure 1: Simulated autocorrelation functions (ACF) of $\ln \Lambda_{0, i}$ and $x_{i}$ based on an underlying Weibull-ACPH(1,0) structure with $\alpha=0.5$ and $p=0.5$. Categorizations based on (simulated) unconditional quantiles of $\ln \Lambda_{0, i}$. Left: 0.5-quantile, middle: 0.25-, 0.5-, 0.75-quantiles, right: 0.1-, $0.2-, \ldots, 0.9$-quantiles. Solid line: ACF of $\ln \Lambda_{0, i}$ based on (15). Broken line: ACF of $\ln \Lambda_{0, i}$ based on (17). Dotted line: ACF of $x_{i}$ implied by (17).
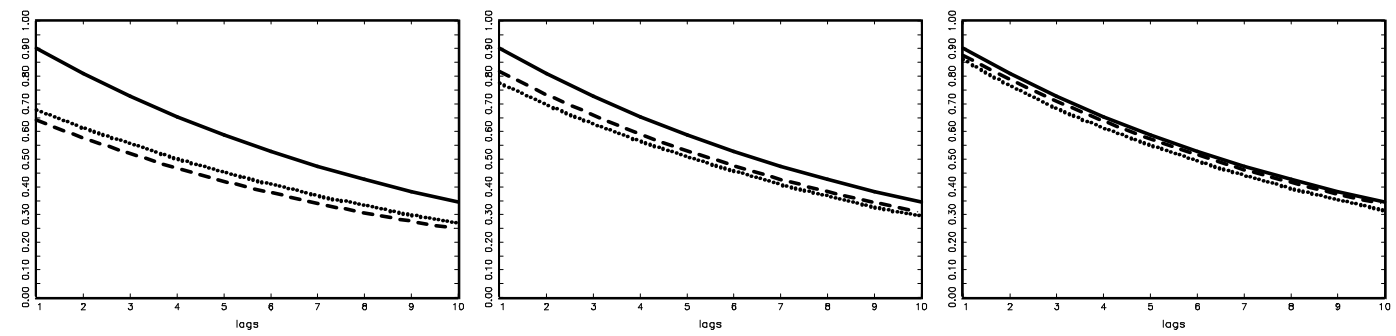

Figure 2: Simulated autocorrelation functions (ACF) of $\ln \Lambda_{0, i}$ and $x_{i}$ based on an underlying Weibull-ACPH(1,0) structure with $\alpha=0.9$ and $p=0.5$. Categorizations based on (simulated) unconditional quantiles of $\ln \Lambda_{0, i}$. Left: 0.5 -quantile, middle: 0.25 -, 0.5-, 0.75-quantiles, right: 0.1-, $0.2-, \ldots, 0.9$-quantiles. Solid line: $\mathrm{ACF}$ of $\ln \Lambda_{0, i}$ based on (15). Broken line: ACF of $\ln \Lambda_{0, i}$ based on (17). Dotted line: ACF of $x_{i}$ implied by (17).

Figures 1 and 2 show the ACF's for all three simulated processes based on underlying $\operatorname{ARMA}(1,0)$ dynamics with parameters $\alpha=0.5$ and $\alpha=0.9$, respectively, based on 100,000 random draws. We observe a close relationship between the autocorrelation functions of the three simulated processes. However, this relation depends rather on the chosen categorization than on the strength

\footnotetext{
${ }^{15}$ We also simulated SACPH processes based on more flexible functional forms for $\lambda_{0}$, however, did not find substantially different results regarding the dynamic properties of the resulting duration process.
} 
of the serial dependence. Whereas for the two-category-grouping clear differences in the autocorrelation functions are observed, quite similar shapes are revealed for the finer categorizations. It is nicely illustrated that the ACF implied by the SACPH process, (17), converges towards the limiting case, eq. (15), when the categorization becomes finer. Moreover, we also observe a quite close relationship between the ACF of the latent variables $\ln \Lambda_{0, i}$ and the implied observable durations $x_{i}$.
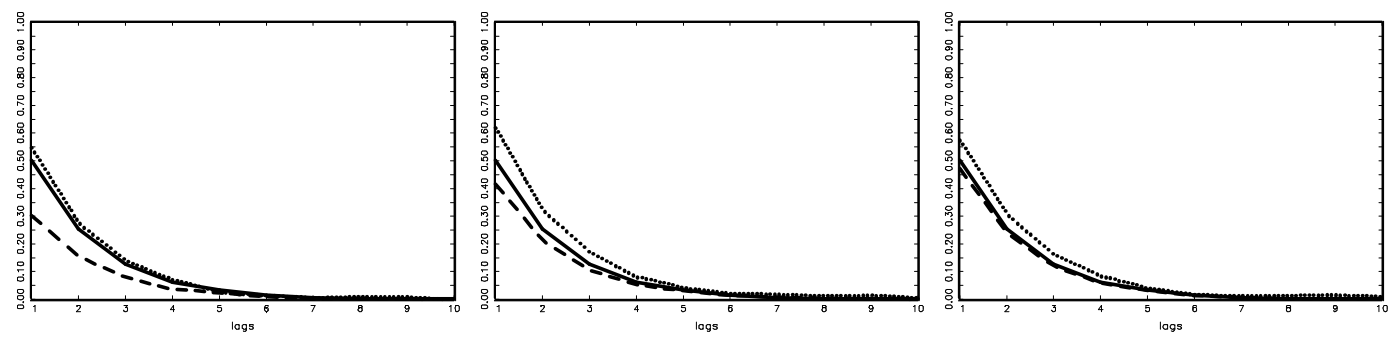

Figure 3: Simulated autocorrelation functions (ACF) of $\ln \Lambda_{0, i}$ and $x_{i}$ based on an underlying Weibull-ACPH(1,1) structure with $\alpha=0.5, \beta=0.7$ and $p=0.5$. Categorizations based on (simulated) unconditional quantiles of $\ln \Lambda_{0, i}$. Left: 0.5-quantile, middle: 0.25-, 0.5-, 0.75-quantiles, right: $0.1-, 0.2-, \ldots, 0.9$-quantiles. Solid line: ACF of $\ln \Lambda_{0, i}$ based on (15). Broken line: ACF of $\ln \Lambda_{0, i}$ based on (17). Dotted line: ACF of $x_{i}$ implied by (17).
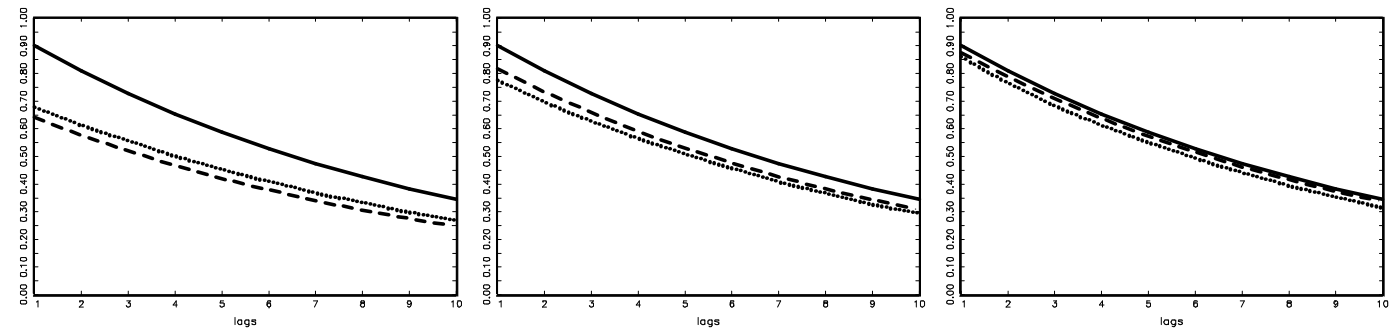

Figure 4: Simulated autocorrelation functions (ACF) of $\ln \Lambda_{0, i}$ and $x_{i}$ based on an underlying Weibull-ACPH(1,1) structure with $\alpha=0.5, \beta=0.5$ and $p=0.5$. Categorizations based on (simulated) unconditional quantiles of $\ln \Lambda_{0, i}$. Left: 0.5-quantile, middle: 0.25-, 0.5-, 0.75-quantiles, right: $0.1-, 0.2-, \ldots, 0.9$-quantiles. Solid line: ACF of $\ln \Lambda_{0, i}$ based on (15). Broken line: ACF of $\ln \Lambda_{0, i}$ based on (17). Dotted line: ACF of $x_{i}$ implied by (17).

These results are confirmed by the simulations based on $\mathrm{SACPH}(1,1)$ processes (Figures 3 and 4). We can conclude that for a sufficiently fine categorization, the autocorrelation structures of the latent and the observable processes are quite similar. Hence, it turns out that the ACF implied by the estimated ARMA coefficients of an SACPH model is a good proxy for the ACF implied by the limiting case, eq. (15), and for the ACF of the resulting observed duration process. This eases the interpretation of the parameters considerably.

\subsection{Evaluating the Estimation Quality in Dependence of the Categorization. The} main advantage of using a dynamic based on generalized errors, eq. (17), instead of a dynamic based on true (however unobservable) errors, eq. (15), are the straightforward and fast maximum likelihood estimation and the fact that the generalized errors $e_{i}$ employed in the approximation are equal to the true errors $\epsilon_{i}^{*}$ if the baseline hazard is known. Yet, as it is at the core of our model that the baseline hazard $\lambda_{0}$ is not known, the question to be answered in this section 
is how large the approximation error is, if the underlying latent errors are approximated by generalized errors on the basis of a given (possibly rough) categorization.

To gain some evidence on the bias incurred by the categorization approach, we perform a Monte Carlo study for different sample sizes and two different categorizations. We assume that the true DGP corresponds to the limiting case, (15), which is simulated using random draws from the standard extreme value distribution. Since the focus is here on the bias of the dynamic parameters, the threshold parameters $\mu_{k}^{*}$ as given by eq. (8) are predetermined and are set to certain quantiles of $\ln \Lambda_{0, i}$. We focus on two alternative categorizations with $K_{1}=2$ and $K_{2}=10$ categories associated with the 0.5 -quantile, as well as the $0.1-, \ldots, 0.9$-quantiles of $\ln \Lambda_{0, i}$, respectively. I.e., as input for the estimation step we use the simulated time series of the predetermined thresholds $\mu_{k}^{*}$ associated with the individual observed categories. Based on these series, we estimate SACPH processes according to (13), (14) and (17). The two-category-model is replicated for two sample sizes $n=50$ and $n=500$. The model with more thresholds is only estimated for a small sample size $n=50$. This set-up allows us to compare the improvement achieved by increasing the number of observations versus the benefit of a better approximation of the baseline hazard.

Table 1: Summary statistics for a Monte Carlo study based on $\operatorname{SACPH}(p, q)$ models with $K$ categories and $n$ observations. MSE denotes the mean squared error and MAE denotes the mean absolute error.

\begin{tabular}{rcccrrr}
\hline $\mathrm{p}$ & $\mathrm{q}$ & $\mathrm{K}$ & $\mathrm{n}$ & bias & MSE & MAE \\
\hline 1 & 0 & 2 & 50 & -0.0062 & 0.0294 & 0.1188 \\
1 & 0 & 10 & 50 & 0.0045 & 0.0096 & 0.0734 \\
1 & 0 & 2 & 500 & 0.0053 & 0.0024 & 0.0372 \\
0 & 1 & 2 & 50 & -0.0079 & 0.0743 & 0.2025 \\
0 & 1 & 10 & 50 & -0.0053 & 0.0189 & 0.0939 \\
0 & 1 & 2 & 500 & 0.0158 & 0.0200 & 0.0959 \\
\hline
\end{tabular}

We consider the cases of $\operatorname{ARMA}(1,0)$ and $\operatorname{ARMA}(0,1)$ dynamics. A range of parameter values for $\alpha$ and $\beta$ are covered in the simulations, concisely, $\alpha, \beta \in \mathcal{Q}=\{-0.9,-0.8, \ldots, 0.8,0.9\}$ providing $n_{i}^{M C}=1,000, i \in \mathcal{Q}$, replications for each value. A summary of the results for all $n^{M C}=19,000$ replications are reported in Table 1 . It gives the aggregated descriptive statistics of the biases, i.e. the differences between the true parameters and the corresponding estimates, $\alpha^{(i)}-\hat{\alpha}^{(i)}$, and $\beta^{(i)}-\hat{\beta}^{(i)}$, for $i=1, \ldots, n^{M C}$. Although we aggregate over all parameter values, the small sample properties match the expectation build from asymptotic theory, i.e. the variance decreases over an increasing sample size. The results indicate that even a moderately sized sample of 50 observations is quite sufficient to obtain reasonable results. In particular, for the $\mathrm{SACPH}(1,0)$ model the asymptotic properties seem to hold quite nicely. The performance of the $\operatorname{SACPH}(0,1)$ model seems to be worse than of the corresponding $\operatorname{SACPH}(1,0)$ model.

To gain more insight into the consequences the categorization grid of the durations bears for the estimation, the results of the Monte Carlo experiment are scrutinized with respect to the parameters of the model, $\alpha$ and $\beta$. Box plots of the biases of $\hat{\alpha}$ for each of the 19 considered 
values of $\alpha$ are given in Figures 5 to 7 . The results are quite encouraging and indicate that for most parameter constellations the bias is very close to zero. Even the quite considerable bias incurred for an $\operatorname{SACPH}(1,0)$ based on $K=2$ categories is reduced substantially once a higher parameterized model based on $K=10$ categories is employed. Furthermore, for a reasonable sample size $(n=500)$ even for the two-category-model, the performance of the estimator is quite encouraging over all parameter values considered.

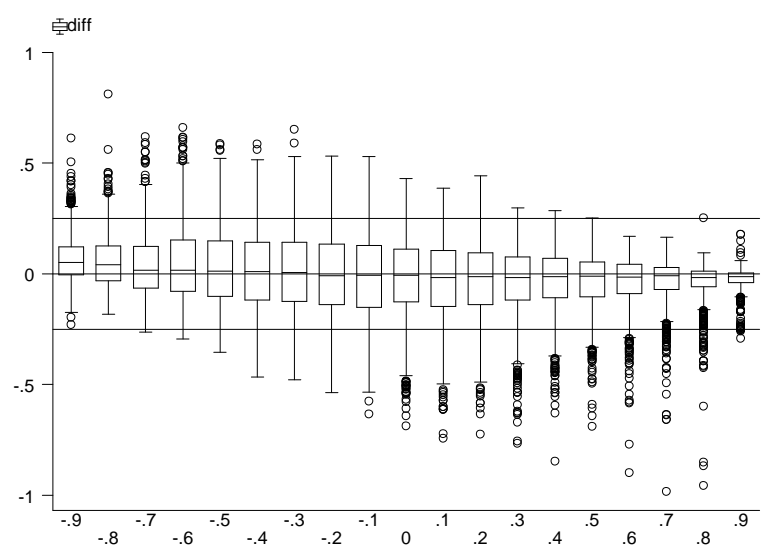

Figure 5: $\operatorname{SACPH}(\mathbf{1}, \mathbf{0}), K=2, n=50$ : Box plots of $\alpha^{(i)}-\hat{\alpha}^{(i)}$ for 19 values of the parameter $\alpha^{(i)}$ in a Monte Carlo study with $n_{i}^{M C}=1,000$ replications for each set $i=1, \ldots, 19$.

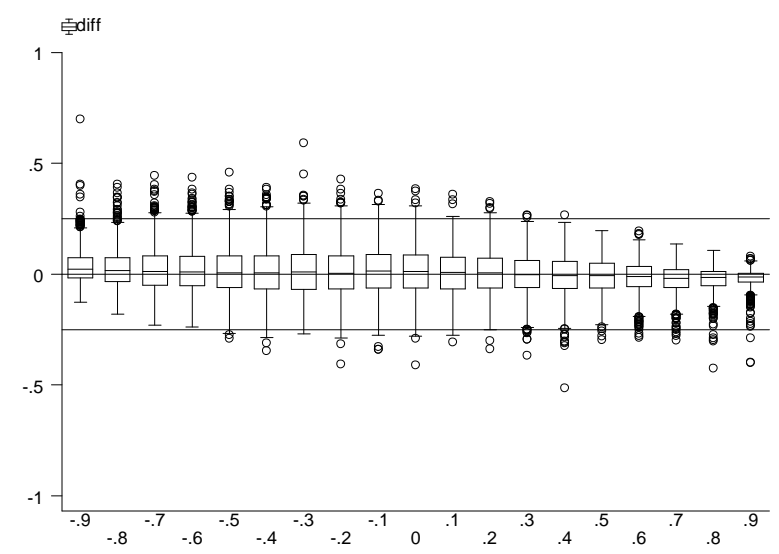

Figure 6: $\operatorname{SACPH}(\mathbf{1}, \mathbf{0}), K=10, n=50$ : Box plots of $\alpha^{(i)}-\hat{\alpha}^{(i)}$ for 19 values of the parameter $\alpha^{(i)}$ in a Monte Carlo study with $n_{i}^{M C}=1,000$ replications for each set $i=1, \ldots, 19$. 


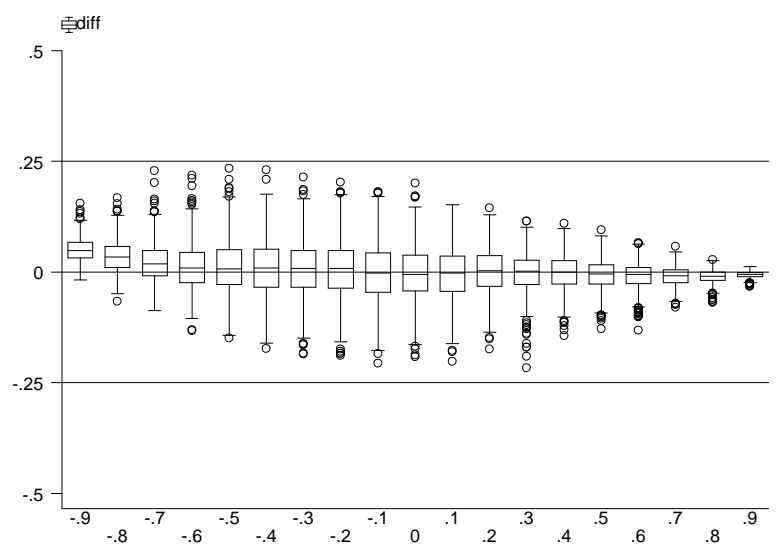

Figure 7: $\operatorname{SACPH}(\mathbf{1}, \mathbf{0}), K=2, n=500$ : Box plots of $\alpha^{(i)}-\hat{\alpha}^{(i)}$ for 19 values of the parameter $\alpha^{(i)}$ in a Monte Carlo study with $n_{i}^{M C}=1,000$ replications for each set $i=1, \ldots, 19$.

Figures 8-10 give the corresponding results for $\operatorname{SACPH}(0,1)$ models. Although, qualitatively similar, it is evident from the study that the $\operatorname{SACPH}(0,1)$ model performs worse than the corresponding $\mathrm{SACPH}(1,0)$ model. After an increase in the number of categories from $K=2$ to $K=10$, the approximation reaches about the quality of the $\operatorname{SACPH}(1,0)$ process with $K=2$ categories, except for the parameter value $\beta=0.9$. The reason for this can be found in the differing $\mathrm{ACF}$ of an $\mathrm{AR}(1)$ and an $\mathrm{MA}(1)$ process. The relatively bad performance of the $\operatorname{SACPH}(0,1)$ process for parameters $\beta$ with a large absolute value is due to the flattening out of the ACF towards the limits of the invertible region.

Overall, we can summarize that for moderate sample sizes and number of categories the bias induced by the categorization approach is rather small and the approximation of true errors by generalized errors is quite good. Hence, if the true DGP corresponds to (15), the SACPH model provides a close approximation to it. If the true DGP is given by (17), the SACPH estimator is consistent anyway.

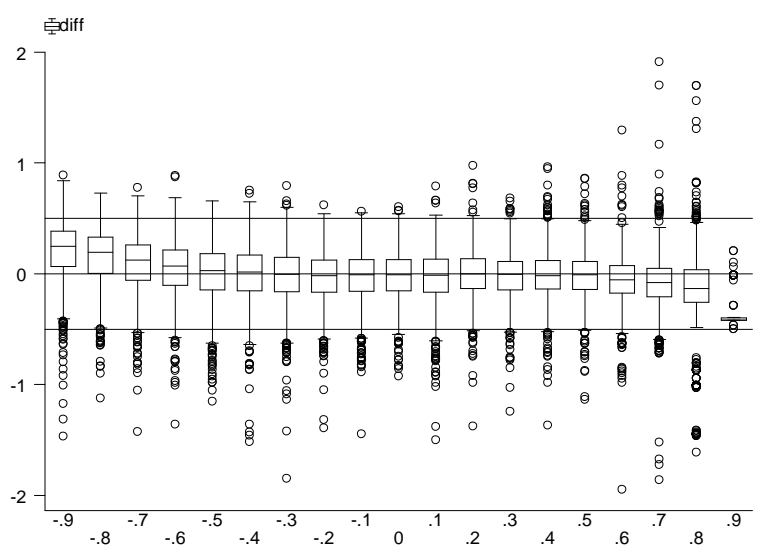

Figure 8: $\mathbf{S A C P H}(\mathbf{0 , 1}), K=2, n=50$ : Box plots of $\beta^{(i)}-\hat{\beta}^{(i)}$ for 19 values of the parameter $\beta^{(i)}$ in a Monte Carlo study with $n_{i}^{M C}=1,000$ replications for each set $i=1, \ldots, 19$. 


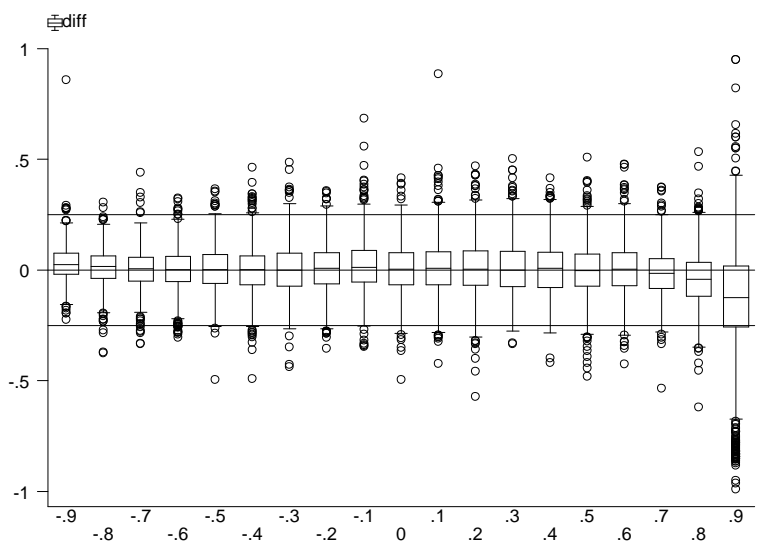

Figure 9: $\operatorname{SACPH}(\mathbf{0}, \mathbf{1}), K=10, n=50$ : Box plots of $\beta^{(i)}-\hat{\beta}^{(i)}$ for 19 values of the parameter $\beta^{(i)}$ in a Monte Carlo study with $n_{i}^{M C}=1,000$ replications for each set $i=1, \ldots, 19$.

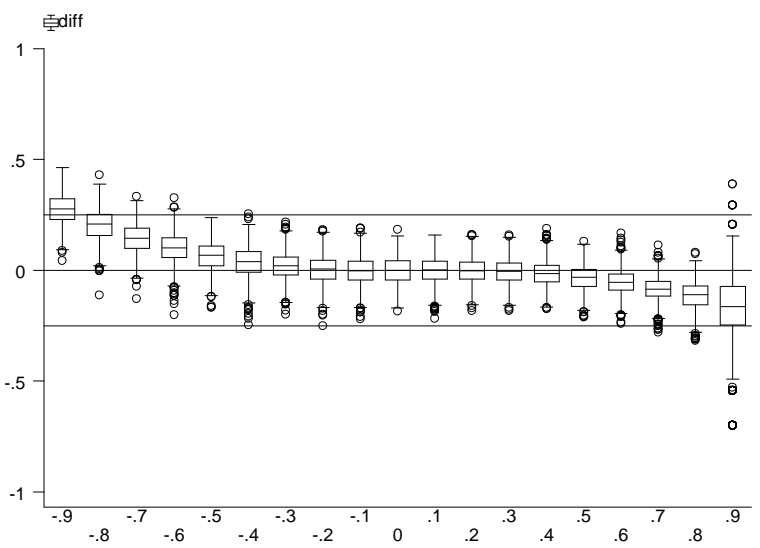

Figure 10: $\operatorname{SACPH}(\mathbf{0 , 1}), K=2, n=500$ : Box plots of $\beta^{(i)}-\hat{\beta}^{(i)}$ for 19 values of the parameter $\beta^{(i)}$ in a Monte Carlo study with $n_{i}^{M C}=1,000$ replications for each set $i=1, \ldots, 19$.

\section{Extensions of the SACPH Model}

5.1. Censoring. A typical property of economic duration data is the occurrence of censoring leading to incomplete spells. A wide strand of econometric duration literature focusses on the consideration of censoring mechanisms ${ }^{16}$. For example, in the context of financial point processes, censoring occurs if there exist intervals in which the point process cannot be observed directly. This might be, for example, due to non-trading periods, like nights, weekends, holidays or trading halts. Assume in the following that it is possible to identify whether a particular point $t_{i}$ lies within such a censoring interval. By referring to our application in Section 7 consider as an example a point process where the points are associated with the occurrence of a cumulative absolute price change of given size. Then, prices move during non-trading periods as well (due to trading on other markets), but can be observed at the earliest at the beginning of the next

\footnotetext{
${ }^{16}$ See, for example, Horowitz and Neumann $(1987,1989)$, the survey by Neumann (1997), Gorgens and Horowitz (1999) or Orbe, Ferreira, and Nunez-Anton (2002).
} 
trading period. In this case, we only know that a price event occurred but we do not know when it exactly has occurred. ${ }^{17}$ Hence, we can identify only the minimum length of the corresponding duration (i.e., the time from the previous point $t_{i-1}$ to the beginning of the censoring interval) and the maximum length of the spell (the time from the end of the censoring interval to the next point $\left.t_{i+1}\right)$.

In the following, $t_{i}^{l}$ and $t_{i}^{u}$ with $t_{i}^{l} \leq t_{i} \leq t_{i}^{u}$ are defined as the boundaries of a potential censoring interval around $t_{i}$, and $c_{i}$ is defined as the indicator variable that indicates whether censoring occurs, i.e.

$$
c_{i}= \begin{cases}1 & \text { if } t_{i} \in\left(t_{i}^{l} ; t_{i}^{u}\right) \\ 0 & \text { if } t_{i}=t_{i}^{l}=t_{i}^{u}\end{cases}
$$

In the case of left-censoring, right-censoring or left-right-censoring, the non-observed duration $x_{i}$ can be only approximated by the boundaries $x_{i} \in\left[x_{i}^{l}, x_{i}^{u}\right]$ where the lower and upper boundary $x_{i}^{l}$ and $x_{i}^{u}$ are computed corresponding to

$$
x_{i} \in\left\{\begin{array}{llll}
{\left[t_{i}-t_{i-1}^{u} ; t_{i}-t_{i-1}^{l}\right]} & \text { if } c_{i-1}=1, c_{i}=0 & \text { (left-censoring) } \\
{\left[t_{i}^{l}-t_{i-1} ; t_{i}^{u}-t_{i-1}\right]} & \text { if } c_{i-1}=0, c_{i}=1 \quad \text { (right-censoring) } \\
{\left[t_{i}^{l}-t_{i-1}^{u} ; t_{i}^{u}-t_{i-1}^{l}\right]} & \text { if } c_{i-1}=1, c_{i}=1 \quad \text { (left-right-censoring). }
\end{array}\right.
$$

A common assumption that is easily fulfilled in the context of financial data is the assumption of independent censoring. This assumption implies that the censoring mechanism is determined exogenously and is not driven by the duration process itself. For a detailed exposition and a discussion of different types of censoring mechanisms, see e.g. Neumann (1997).

Under the assumption of independent censoring, the likelihood can be decomposed into

$$
\mathcal{L}\left(W ; \theta \mid c_{1}, \ldots, c_{n}\right)=\mathcal{L}\left(W ; \theta, c_{1}, \ldots, c_{n}\right) \cdot \mathcal{L}\left(c_{1}, \ldots, c_{n}\right) .
$$

Define in the following $x_{i}^{d, l}$ and $x_{i}^{d, u}$ as the categorized counterparts to the duration boundaries $x_{i}^{l}$ and $x_{i}^{u}$. Hence, $x_{i}^{d} \in\left[x_{i}^{d, l}, x_{i}^{d, u}\right]$, where $x_{i}^{d, l}$ and $x_{i}^{d, u}$ are computed corresponding to eq. (23) by accounting for the observation rule, eq. (9).

Then, in the case of a censored observation $i$, the corresponding log likelihood contribution in eq. (12) is replaced by

$$
\operatorname{Pr}\left[x_{i}^{l} \leq x_{i}^{d} \leq x_{i}^{u} \mid \mathcal{F}_{i-1}^{d}, c_{i-1}, c_{i}, c_{i+1}\right]=F_{\epsilon^{*}}\left(\mu^{* u}-\phi_{i}\right)-F_{\epsilon^{*}}\left(\mu^{* l}-\phi_{i}\right),
$$

where

$$
\begin{array}{ll}
\mu^{* l}=\mu_{k}^{*} & \text { if } x_{i}^{d, l}=k+1, \\
\mu^{* u}=\mu_{k}^{*} & \text { if } x_{i}^{d, u}=k .
\end{array}
$$

\footnotetext{
${ }^{17}$ However, note that we cannot identify whether more than one price movement have occurred during the non-trading period.
} 
Moreover, the computation of the generalized error needs to be slightly modified. Hence, in the case of censoring, the conditional expectation of $\epsilon_{i}^{*}$ is computed as

$$
\begin{aligned}
e_{i} & =\mathrm{E}\left[\epsilon_{i}^{*} \mid x_{i}^{d}, \phi_{i}, c_{i-1}, c_{i}, c_{i+1}\right]=\mathrm{E}\left[\epsilon_{i}^{*} \mid x_{i}^{d, l}, x_{i}^{d, u}, \phi_{i}\right] \\
& =\frac{\kappa\left(\nu_{i}^{l}, \nu_{i}^{u}\right)}{F_{\epsilon^{*}}\left(\nu_{i}^{l}\right)-F_{\epsilon^{*}}\left(\nu_{i}^{u}\right)},
\end{aligned}
$$

where $\nu_{i}^{l}:=\mu^{* l}-\phi_{i}$ and $\nu_{i}^{u}:=\mu^{* u}-\phi_{i}$.

5.2. Unobserved Heterogeneity. A further advantage of the SACPH model is that it is readily extended to allow for unobservable heterogeneity. In duration literature, it is well known that ignoring unobserved heterogeneity can lead to biased estimates of the baseline hazard function. ${ }^{18}$ Following Han and Hausman (1990), we specify unobserved heterogeneity effects by a random variable which enters the hazard function multiplicatively leading to a mixed SACPH model. From an econometric point of view, accounting for unobserved heterogeneity can be interpreted as an additional degree of freedom. Lancaster (1997) illustrates that the inclusion of a heterogeneity variable can capture errors in the variables. In financial duration data, unobservable heterogeneity effects can be associated with different states of the market.

The standard procedure to account for unobserved heterogeneity in the SACPH model is to introduce an i.i.d. random variable $v_{i}$ in specification (18) to obtain

$$
\lambda\left(x ; \mathcal{F}_{i-1}^{d}, v_{i}\right)=\lambda_{0}(x) \cdot v_{i} \cdot \exp \left(-\phi_{i}\right) .
$$

For the random variable $v_{i}$ we assume a Gamma distribution with mean one and variance $\eta^{-1}$, which is standard for this type of mixture models, see e.g. Lancaster (1997). Then, the c.d.f. of the compounded model is obtained by integrating out $v_{i}$ leading to

$$
F\left(x_{i} ; \mathcal{F}_{i-1}^{d}\right)=1-\left[1+\eta^{-1} \exp \left(-\phi_{i}\right) \Lambda_{0, i}\right]^{-\eta} .
$$

Note that this is identical to the c.d.f of a $\operatorname{BurrII}(\eta)$ distribution under appropriate parameterization. The latter gives rise to an analogue model based on the categorization approach outlined in Section 3. By augmenting the log linear model of the integrated baseline hazard by the compounder, we obtain an extended SACPH model based on the modified latent process

$$
\ln \Lambda_{0, i}=\ln (\eta)+\phi_{i}+\epsilon_{i}^{*},
$$

where the error term $\epsilon_{i}^{*}$ follows a $\operatorname{BurrII}(\eta)$ distribution with p.d.f. and c.d.f. given by

$$
\begin{aligned}
f_{\epsilon^{*}}(x) & =\frac{\eta \exp (x)}{[1+\exp (x)]^{\eta+1}}, \\
F_{\epsilon^{*}}(x) & =1-[1+\exp (x)]^{-\eta} .
\end{aligned}
$$

It is easily shown that

$$
\lim _{\eta \rightarrow \infty}\left[1+\eta^{-1} \phi_{i} \Lambda_{0, i}\right]^{-\eta}=\exp \left(-\Lambda_{0, i} \phi_{i}\right)
$$

\footnotetext{
${ }^{18}$ See e.g. Lancaster (1979) or Heckmann and Singer (1984).
} 
i.e. for $\eta^{-1}=\operatorname{Var}\left(v_{i}\right) \rightarrow 0$, the $\operatorname{BurrII}(\eta)$ distribution converges to the standard extreme value distribution. Hence, if no unobservable heterogeneity effects exist, the model coincides with the basic SACPH model.

The estimation procedure is similar to the procedure described in Section 3. The difference is just that the model is now based on a $\operatorname{BurrII}(\eta)$ distribution. Apart from an obvious adjustment to the generalized errors, the relationship between the estimated thresholds and the baseline survivor function, as given in eq. (11), is slightly modified to

$$
S_{0}\left(\bar{x}_{k}\right)=\frac{1}{\left[1+\exp \left(\mu_{k}^{*}-\ln (\eta)\right)\right]^{\eta}}, \quad k=1, \ldots, K-1 .
$$

\section{Testing the SACPH Model}

An obvious way to test for correct specification of the SACPH model is to evaluate the properties of the series of the estimated $\log$ integrated hazard functions $\hat{\epsilon}_{i}^{*}=\ln \hat{\Lambda}\left(x_{i}\right)$ which should be i.i.d. standard extreme value distributed or $\operatorname{BurrII}(\eta)$ distributed, respectively. However, the difficulty is that we cannot directly estimate $\epsilon_{i}^{*}$ but only its conditional expectation $\hat{e}_{i}=\hat{\mathrm{E}}\left[\epsilon_{i}^{*} \mid \mathcal{F}_{i}^{d}\right]$. Thus, the SACPH model has to be evaluated by comparing the distributional and dynamical properties of $\hat{e}_{i}$ with their theoretical counterparts.

The unconditional mean of $e_{i}$ is straightforwardly computed as

$$
\mathrm{E}\left[e_{i}\right]=\mathrm{E}\left[\mathrm{E}\left[\epsilon_{i}^{*} \mid x_{i}^{d}, \phi_{i}\right]\right]=\mathrm{E}\left[\epsilon_{i}^{*}\right]
$$

However, the computation of higher order moments of $e_{i}$ is a difficult task. The reason is that the categorization is based on $\ln \Lambda_{0, i}$, and thus the category boundaries for $\epsilon_{i}^{*}, \nu_{i, k}=\mu_{k}^{*}-\phi_{i}$, are time-varying and depend itself on lags of $e_{i}$. Therefore, the derivation of theoretical moments can only be performed on the basis of the estimated model dynamics, and thus they are of limited value for powerful diagnostic checks of the model. Hence, only upper limits for the moments in the limiting case $K \rightarrow \infty$ can be given. In this case, $e_{i}=\epsilon_{i}^{*}$, and thus the moments of $e_{i}$ correspond to the moments of the standard extreme value or $\operatorname{BurrII}(\eta)$ distribution, respectively.

Nevertheless, the dynamic properties of the $\hat{e}_{i}$ series is evaluated based on a test for serial dependence as proposed by Gourieroux, Monfort, and Trognon (1985). The test is based on the direct relationship between the score of the observable and the latent model. By considering a model which includes unobserved heterogeneity (see Section 5.2), the latent model can be rewritten in a more general form as

$$
\begin{aligned}
\ln \Lambda_{0, i} & =\ln (\eta)+\phi_{i}+u_{i}, \\
u_{i} & =\alpha_{j} u_{i-j}+\epsilon_{i}^{*},
\end{aligned}
$$


where $\epsilon_{i}^{*}$ is i.i.d. $\operatorname{BurrII}(\eta)$ distributed and $j$ denotes the tested lag. As shown in the appendix, a $\chi^{2}$ statistic for the null hypothesis of no serial dependence, $H_{0}: \alpha_{j}=0$, is obtained by

$$
\Upsilon^{(j)}=\frac{\left[\sum_{i=j+1}^{n} \hat{e}_{i-j}\left[1-(\hat{\eta}+1) \hat{\tilde{e}}_{i}\right]\right]^{2}}{\sum_{i=j+1}^{n} \hat{e}_{i-j}^{2}\left[1-(\hat{\eta}+1) \hat{\tilde{e}}_{i}\right]^{2}} \stackrel{a}{\sim} \chi^{2}(1),
$$

where $\tilde{e}_{i}:=\mathrm{E}\left[\frac{\exp \left(\epsilon_{i}^{*}\right)}{1+\exp \left(\epsilon_{i}^{*}\right)} \mid \mathcal{F}_{i}^{d}\right]$. Correspondingly, for the standard extreme value case, the test modifies to

$$
\Upsilon^{(j)}=\frac{\left[\sum_{i=j+1}^{n} \hat{e}_{i-j}\left(\hat{\tilde{e}}_{i}-1\right)\right]^{2}}{\sum_{i=j+1}^{n} \hat{e}_{i-j}^{2}\left[\hat{\tilde{e}}_{i}-1\right]^{2}} \stackrel{a}{\sim} \chi^{2}(1)
$$

with $\tilde{e}_{i}:=\mathrm{E}\left[\exp \left(\epsilon_{i}^{*}\right) \mid \mathcal{F}_{i}^{d}\right]$

\section{Applying And Evaluating the SACPH Model}

7.1. Estimating Conditional Price Change Probabilities. In this section, we illustrate an application of the SACPH model to the modelling of price durations, i.e. the time it takes for the price of an asset to leave an interval of a given size. Hence, in contrast to classical approaches where one aggregates over time, here, the aggregation scheme is based on the price process itself. Engle and Russell (1998) and Gerhard and Hautsch (2002) illustrate that price durations are strongly related to return volatility and allow to construct alternative risk measures that explicitly account for time structures in the price process. Price durations allow for the quantification of the risk of a given price change within a certain time interval and are of particular interest whenever an investor is able to determine his risk in terms of a certain price movement.

In the following analysis, we estimate the conditional probability to observe a cumulative absolute price change of predetermined size within a certain time. In this context, we explicitly focus on very large price movements which might be of interest for short-term risk management and can be associated with the execution probability of a hypothetical limit order. Our data base consists of a time series of transaction data originating from the trading process of the German Bund future traded at the electronic trading system of the EUREX. The sample consists of 883 trading days between $04 / 03 / 94$ and $05 / 03 / 97$, corresponding to 2,072,785 individual transactions. The Bund future is a future contract on a synthetical $6 \%$ German government bond. It is one the most liquid future contracts in Europe and within the analyzed sample period it had a face value of DEM 250,000. It has a maturity of 8.5 years and four contract maturities per year, March, June, September and December. Prices are denoted in basis points of face value, thus, in the considered sampling interval one tick was equivalent to a value of DEM 25. In the following we focus on durations associated with cumulative absolute price changes of 
30, 40 and 50 ticks lasting 24, 44 and 66 hours on average resulting in 1226, 698 and 484 price duration observations, respectively, over the complete sample period (see Table 2).

Table 2: Distribution of categorized non-censored price durations and lower boundaries of censored price durations, as well as the number of censored observations. Based on Bund future price durations using 30, 40 and 50 tick price changes. Descriptive statistics given in hours.

\begin{tabular}{|c|c|c|c|c|c|c|c|c|c|}
\hline & \multicolumn{3}{|c|}{30 ticks } & \multicolumn{3}{|c|}{40 ticks } & \multicolumn{3}{|c|}{50 ticks } \\
\hline & obs & mean & cens & obs & mean & cens & obs & mean & cens \\
\hline$[0 h, 0.5 h]$ & 67 & 0.287 & 11 & 13 & 0.300 & 6 & 3 & 0.361 & 2 \\
\hline$[0.5 h, 1 h]$ & 82 & 0.771 & 24 & 20 & 0.778 & 9 & 4 & 0.733 & 4 \\
\hline$(1 h, 2 h]$ & 118 & 1.380 & 27 & 37 & 1.493 & 12 & 23 & 1.430 & 11 \\
\hline$(2 h, 3 h]$ & 72 & 2.494 & 22 & 32 & 2.444 & 7 & 17 & 2.382 & 4 \\
\hline$(3 h, 4 h]$ & 58 & 3.528 & 7 & 19 & 3.533 & 2 & 10 & 3.621 & 2 \\
\hline$(4 h, 5 h]$ & 39 & 4.581 & 6 & 22 & 4.445 & 4 & 9 & 4.564 & 2 \\
\hline$(5 h, 6 h]$ & 48 & 5.465 & 3 & 20 & 5.516 & 4 & 10 & 5.435 & 5 \\
\hline$(6 h, 7 h]$ & 40 & 6.506 & 6 & 27 & 6.511 & 3 & 18 & 6.409 & 2 \\
\hline$(7 h, 8 h]$ & 30 & 7.484 & 7 & 19 & 7.554 & 5 & 11 & 7.571 & 2 \\
\hline$(8 h, 9 h]$ & 19 & 8.392 & 17 & 8 & 8.465 & 9 & 10 & 8.308 & 4 \\
\hline$(9 h, 24 h]$ & 174 & 19.403 & 19 & 81 & 19.829 & 13 & 43 & 20.115 & 3 \\
\hline$(24 h, 28 h]$ & 58 & 25.429 & 7 & 48 & 25.747 & 4 & 24 & 25.355 & 4 \\
\hline$(28 h, 32 h]$ & 38 & 29.932 & 1 & 26 & 30.397 & 6 & 25 & 29.968 & 3 \\
\hline$(24 h, 36 h]$ & 2 & 32.583 & 8 & 1 & 32.204 & 6 & 0 & - & 2 \\
\hline$(36 h, 48 h]$ & 25 & 43.683 & 0 & 25 & 44.041 & 0 & 28 & 43.318 & 0 \\
\hline$(48 h, 72 h]$ & 60 & 61.141 & 7 & 62 & 61.445 & 5 & 43 & 59.700 & 4 \\
\hline$(72 h, 96 h]$ & 51 & 84.360 & 6 & 38 & 86.217 & 4 & 27 & 85.671 & 5 \\
\hline$(96 h, 120 h]$ & 26 & 108.997 & 4 & 35 & 105.171 & 4 & 27 & 107.722 & 3 \\
\hline$(120 h, 144 h]$ & 18 & 129.724 & 2 & 9 & 133.271 & 7 & 20 & 131.571 & 1 \\
\hline$(144 h, 168 h]$ & 5 & 157.528 & 1 & 15 & 155.533 & 5 & 22 & 154.114 & 1 \\
\hline$(168 h, 192 h]$ & 6 & 171.227 & 0 & 6 & 175.071 & 3 & 12 & 175.733 & 2 \\
\hline$(192 h, 240 h]$ & 1 & 221.664 & 1 & 5 & 220.328 & 0 & 10 & 213.893 & 2 \\
\hline$(240 h, 288 h]$ & 2 & 253.001 & 0 & 7 & 259.383 & 0 & 7 & 255.332 & 0 \\
\hline$(288 h, \infty$ & 1 & 361.949 & 0 & 5 & 312.421 & 0 & 11 & 317.666 & 2 \\
\hline Total & 1040 & 23.9468 & 186 & 580 & 43.8744 & 118 & 414 & 65.8607 & 70 \\
\hline
\end{tabular}

For the resulting price durations, we choose a categorization scheme which ensures sufficient frequencies in each category and includes durations up to 240 hours for 30 and 40 price changes and up to 288 hours for 50 tick price changes. Table 2 shows the distribution of the categorized durations for the individual price duration series. An important feature of such high aggregated financial duration data is the occurrence of censoring structures. As motivated in Section 5.2, this is due to the fact that price change events can also be caused by news occurring during non-trading periods, like nights, weekends or holidays. Then, the resulting price changes are observable only at the beginning of the next trading day, and thus, it is difficult to identify whether the price changes are driven by overnight news or by information which occurred recently. Following Gerhard and Hautsch (2002), we define the first price event which occurs within the first 15 minutes of a trading day as censored, i.e. this price change is defined to be driven by events occurring overnight. This identification rule implies that price events occurring after the first 15 minutes of a trading day are assumed to be driven by recent information. For these observations, the exact duration is measured as the time since the last observation of the previous trading day. By applying this identification scheme, we obtain 186, 118 and 70 censored observations, respectively, for the three duration series. 
Table 3: ML estimates of SACPH $(1,1)$ models for Bund futures price durations using 30 tick price changes. Standard errors computed based on robust estimates of the covariance matrix. Diagnostics: Log Likelihood (LL), as well as test statistics for a test on serial correlation (inclusive p-values) of the SACPH residuals.

\begin{tabular}{|c|c|c|c|c|c|c|c|c|c|c|}
\hline & \multicolumn{2}{|c|}{ (1) } & \multicolumn{2}{|c|}{ (2) } & \multicolumn{2}{|c|}{ (3) } & \multicolumn{2}{|c|}{ (4) } & \multicolumn{2}{|c|}{ (5) } \\
\hline & est. & S.E. & est. & S.E. & est. & S.E. & est. & S.E. & est. & S.E. \\
\hline \multicolumn{11}{|c|}{ Thresholds } \\
\hline$\nu_{1}^{*}\left(\bar{x}_{1}=0.5 h\right)$ & -1.325 & 0.800 & & & & & & & & \\
\hline$\nu_{1}^{*}\left(\bar{x}_{1}=1 h\right)$ & -0.360 & 0.796 & -0.104 & 0.642 & & & & & & \\
\hline$\nu_{2}^{*}\left(\bar{x}_{2}=2 h\right)$ & 0.485 & 0.810 & 0.737 & 0.657 & & & & & & \\
\hline$\nu_{3}^{*}\left(\bar{x}_{3}=3 h\right)$ & 0.900 & 0.820 & 1.154 & 0.668 & & & & & & \\
\hline$\nu_{4}^{*}\left(\bar{x}_{4}=4 h\right)$ & 1.204 & 0.830 & 1.460 & 0.677 & 1.527 & 0.719 & & & & \\
\hline$\nu_{5}^{*}\left(\bar{x}_{5}=5 h\right)$ & 1.401 & 0.838 & 1.659 & 0.681 & & & & & & \\
\hline$\nu_{6}^{*}\left(\bar{x}_{6}=6 h\right)$ & 1.638 & 0.845 & 1.898 & 0.690 & & & & & & \\
\hline$\nu_{7}^{*}\left(\bar{x}_{7}=7 h\right)$ & 1.834 & 0.851 & 2.097 & 0.697 & & & & & & \\
\hline$\nu_{8}^{*}\left(\bar{x}_{8}=8 h\right)$ & 1.983 & 0.856 & 2.248 & 0.704 & 2.299 & 0.741 & & & & \\
\hline$\nu_{9}^{*}\left(\bar{x}_{9}=9 h\right)$ & 2.083 & 0.859 & 2.349 & 0.709 & & & & & & \\
\hline$\nu_{10}^{*}\left(\bar{x}_{10}=24 h\right)$ & 3.177 & 0.909 & 3.463 & 0.761 & 3.476 & 0.784 & 5.364 & 1.401 & 5.295 & 1.380 \\
\hline$\nu_{11}^{*}\left(\bar{x}_{10}=28 h\right)$ & 3.519 & 0.928 & & & & & & & & \\
\hline$\nu_{12}^{*}\left(\bar{x}_{10}=32 h\right)$ & 3.775 & 0.944 & & & & & & & & \\
\hline$\nu_{13}^{*}\left(\bar{x}_{11}=36 h\right)$ & 3.790 & 0.945 & 4.122 & 0.804 & 4.120 & 0.817 & & & & \\
\hline$\nu_{14}^{*}\left(\bar{x}_{12}=48 h\right)$ & 3.995 & 0.958 & 4.326 & 0.820 & 4.320 & 0.830 & 6.229 & 1.462 & & \\
\hline$\nu_{15}^{*}\left(\bar{x}_{13}=72 h\right)$ & 4.636 & 1.004 & 4.967 & 0.875 & 4.943 & 0.872 & 6.865 & 1.512 & 6.807 & 1.490 \\
\hline$\nu_{16}^{*}\left(\bar{x}_{14}=96 h\right)$ & 5.415 & 1.072 & 5.772 & 0.955 & 5.724 & 0.935 & 7.670 & 1.589 & & \\
\hline$\nu_{17}^{*}\left(\bar{x}_{15}=120 h\right)$ & 6.141 & 1.136 & 6.526 & 1.050 & 6.455 & 1.011 & 8.425 & 1.682 & 8.385 & 1.657 \\
\hline$\nu_{18}^{*}\left(\bar{x}_{16}=144 h\right)$ & 7.107 & 1.249 & 7.531 & 1.188 & 7.428 & 1.120 & 9.429 & 1.803 & & \\
\hline$\nu_{19}^{*}\left(\bar{x}_{17}=168 h\right)$ & 7.620 & 1.322 & 8.066 & 1.261 & 7.945 & 1.179 & 9.962 & 1.859 & 9.928 & 1.827 \\
\hline$\nu_{20}^{*}\left(\bar{x}_{18}=192 h\right)$ & 8.655 & 1.508 & 9.144 & 1.416 & 8.984 & 1.308 & 11.048 & 1.978 & & \\
\hline$\nu_{21}^{*}\left(\bar{x}_{19}=240 h\right)$ & 9.045 & 1.608 & 9.551 & 1.485 & 9.372 & 1.368 & 11.456 & 2.022 & 11.524 & 2.007 \\
\hline$\alpha_{1}$ & 0.985 & 0.005 & 0.984 & 0.009 & 0.986 & 0.007 & 0.990 & 0.004 & 0.990 & 0.004 \\
\hline$\beta_{1}$ & -0.913 & 0.015 & -0.911 & 0.028 & -0.909 & 0.023 & -0.910 & 0.018 & -0.909 & 0.019 \\
\hline$\eta$ & 0.801 & 0.099 & 0.768 & 0.106 & 0.791 & 0.088 & 0.755 & 0.104 & 0.757 & 0.103 \\
\hline Obs & \multicolumn{2}{|c|}{1226} & \multicolumn{2}{|c|}{1226} & \multicolumn{2}{|c|}{1226} & \multicolumn{2}{|c|}{1226} & \multicolumn{2}{|c|}{1226} \\
\hline $\mathrm{LL}$ & \multicolumn{2}{|c|}{-2966} & \multicolumn{2}{|c|}{-2781} & \multicolumn{2}{|c|}{-1967} & \multicolumn{2}{|c|}{-1132} & \multicolumn{2}{|c|}{-944} \\
\hline & est. & p-val. & est. & p-val. & est. & p-val. & est. & p-val. & est. & p-val. \\
\hline$\Upsilon^{(1)}$ & 4.640 & 0.031 & 4.476 & 0.034 & 3.578 & 0.058 & 1.036 & 0.308 & 2.971 & 0.084 \\
\hline$\Upsilon^{(2)}$ & 1.569 & 0.210 & 1.547 & 0.213 & 1.911 & 0.166 & 1.302 & 0.253 & 1.017 & 0.313 \\
\hline$\Upsilon^{(3)}$ & 0.823 & 0.364 & 0.824 & 0.363 & 1.051 & 0.305 & 2.527 & 0.111 & 1.535 & 0.215 \\
\hline$\Upsilon^{(4)}$ & 0.152 & 0.696 & 0.098 & 0.753 & 0.669 & 0.413 & 2.154 & 0.142 & 2.161 & 0.141 \\
\hline$\Upsilon^{(5)}$ & 0.662 & 0.415 & 0.745 & 0.387 & 0.315 & 0.574 & 0.171 & 0.678 & 0.010 & 0.919 \\
\hline
\end{tabular}

Tables 3 through 5 show the estimation results based on SACPH models which account for unobserved heterogeneity. The lag order is chosen according to the Bayes Information Criterion (BIC) indicating an $\operatorname{SACPH}(1,1)$ model as the best specification in all cases. ${ }^{19}$ We find highly significant autoregressive parameters with estimates of $\alpha_{1}$ close to one and estimates of $\beta_{1}$ around -0.9 . This finding indicates a relatively strong persistence in the data and is typical for financial duration series. For the heterogeneity parameter $\eta$, we observe highly significant estimates, as well. Comparisons with re-estimates of the individual models based on SACPH models without unobserved heterogeneity effects (which are not shown here) indicate a clear increase of the maximized log likelihood function and the BIC value when unobserved heterogeneity is taken into account. These findings illustrate the usefulness of the gamma compounded model.

\footnotetext{
${ }^{19}$ We also re-estimated all models by accounting explicitly for possible intraday and time-to-maturity seasonalities based on flexible Fourier forms (see Gerhard and Hautsch, 2002). However, insignificant coefficients and no improvements of the BIC indicated that on such high aggregated levels deterministic seasonality structures seem to be negligible. For this reason we refrain from showing these estimates here. Nevertheless, they are available upon request from the authors.
} 
Table 4: ML estimates of SACPH(1,1) models for Bund future price durations using 40 tick price changes. Standard errors computed based on robust estimates of the covariance matrix. Diagnostics: Log Likelihood (LL), as well as test statistics for a test on serial correlation (inclusive p-values) of the SACPH residuals.

\begin{tabular}{|c|c|c|c|c|c|c|c|c|c|c|}
\hline & \multicolumn{2}{|c|}{ (1) } & \multicolumn{2}{|c|}{$(2)$} & \multicolumn{2}{|c|}{$(3)$} & \multicolumn{2}{|c|}{ (4) } & \multicolumn{2}{|c|}{ (5) } \\
\hline & est. & S.E. & est. & S.E. & est. & S.E. & est. & S.E. & est. & S.E. \\
\hline \multicolumn{11}{|c|}{ Thresholds } \\
\hline$\nu_{1}^{*}\left(\bar{x}_{1}=0.5 h\right)$ & -3.519 & 0.741 & & & & & & & & \\
\hline$\nu_{1}^{*}\left(\bar{x}_{1}=1 h\right)$ & -2.536 & 0.729 & -2.478 & 0.738 & & & & & & \\
\hline$\nu_{2}^{*}\left(\bar{x}_{2}=2 h\right)$ & -1.677 & 0.732 & -1.628 & 0.742 & & & & & & \\
\hline$\nu_{3}^{*}\left(\bar{x}_{3}=3 h\right)$ & -1.207 & 0.734 & -1.159 & 0.745 & & & & & & \\
\hline$\nu_{4}^{*}\left(\bar{x}_{4}=4 h\right)$ & -0.981 & 0.737 & -0.934 & 0.747 & -0.888 & 0.458 & & & & \\
\hline$\nu_{5}^{*}\left(\bar{x}_{5}=5 h\right)$ & -0.744 & 0.742 & -0.698 & 0.753 & & & & & & \\
\hline$\nu_{6}^{*}\left(\bar{x}_{6}=6 h\right)$ & -0.545 & 0.747 & -0.499 & 0.758 & & & & & & \\
\hline$\nu_{7}^{*}\left(\bar{x}_{7}=7 h\right)$ & -0.297 & 0.752 & -0.252 & 0.763 & & & & & & \\
\hline$\nu_{8}^{*}\left(\bar{x}_{8}=8 h\right)$ & -0.130 & 0.757 & -0.085 & 0.768 & -0.034 & 0.459 & & & & \\
\hline$\nu_{9}^{*}\left(\bar{x}_{9}=9 h\right)$ & -0.058 & 0.759 & -0.013 & 0.771 & & & & & & \\
\hline$\nu_{10}^{*}\left(\bar{x}_{10}=24 h\right)$ & 0.743 & 0.792 & 0.789 & 0.805 & 0.819 & 0.474 & 1.231 & 0.778 & 1.276 & 0.788 \\
\hline$\nu_{11}^{*}\left(\bar{x}_{10}=28 h\right)$ & 1.092 & 0.809 & & & & & & & & \\
\hline$\nu_{12}^{*}\left(\bar{x}_{10}=32 h\right)$ & 1.298 & 0.820 & & & & & & & & \\
\hline$\nu_{13}^{*}\left(\bar{x}_{11}=36 h\right)$ & 1.307 & 0.821 & 1.383 & 0.836 & 1.412 & 0.487 & & & & \\
\hline$\nu_{14}^{*}\left(\bar{x}_{12}=48 h\right)$ & 1.527 & 0.834 & 1.598 & 0.849 & 1.626 & 0.491 & 2.043 & 0.794 & & \\
\hline$\nu_{15}^{*}\left(\bar{x}_{13}=72 h\right)$ & 2.177 & 0.876 & 2.231 & 0.893 & 2.258 & 0.505 & 2.680 & 0.808 & 2.746 & 0.822 \\
\hline$\nu_{16}^{*}\left(\bar{x}_{14}=96 h\right)$ & 2.643 & 0.909 & 2.700 & 0.927 & 2.726 & 0.519 & 3.156 & 0.824 & & \\
\hline$\nu_{17}^{*}\left(\bar{x}_{15}=120 h\right)$ & 3.253 & 0.961 & 3.312 & 0.981 & 3.337 & 0.537 & 3.775 & 0.843 & 3.854 & 0.859 \\
\hline$\nu_{18}^{*}\left(\bar{x}_{16}=144 h\right)$ & 3.482 & 0.985 & 3.543 & 1.006 & 3.567 & 0.545 & 4.008 & 0.850 & & \\
\hline$\nu_{19}^{*}\left(\bar{x}_{17}=168 h\right)$ & 4.043 & 1.044 & 4.107 & 1.067 & 4.129 & 0.571 & 4.577 & 0.874 & 4.675 & 0.891 \\
\hline$\nu_{20}^{*}\left(\bar{x}_{18}=192 h\right)$ & 4.403 & 1.094 & 4.469 & 1.119 & 4.491 & 0.592 & 4.942 & 0.889 & & \\
\hline$\nu_{21}^{*}\left(\bar{x}_{19}=240 h\right)$ & 4.810 & 1.160 & 4.878 & 1.187 & 4.899 & 0.623 & 5.355 & 0.912 & 5.452 & 0.932 \\
\hline$\alpha_{1}$ & 0.978 & 0.011 & 0.977 & 0.011 & 0.979 & 0.012 & 0.981 & 0.011 & 0.980 & 0.011 \\
\hline$\beta_{1}$ & -0.891 & 0.026 & -0.892 & 0.025 & -0.894 & 0.023 & -0.881 & 0.022 & -0.877 & 0.023 \\
\hline$\eta$ & 0.905 & 0.127 & 0.897 & 0.130 & 0.899 & 0.056 & 0.879 & 0.063 & 0.874 & 0.064 \\
\hline Obs & \multicolumn{2}{|c|}{698} & \multicolumn{2}{|c|}{698} & \multicolumn{2}{|c|}{698} & \multicolumn{2}{|c|}{698} & \multicolumn{2}{|c|}{698} \\
\hline LL & \multicolumn{2}{|c|}{-1864} & \multicolumn{2}{|c|}{-1774} & \multicolumn{2}{|c|}{-1432} & \multicolumn{2}{|c|}{-1015} & \multicolumn{2}{|c|}{-815} \\
\hline & est. & p-val. & est. & p-val. & est. & p-val. & est. & p-val. & est. & p-val. \\
\hline$\Upsilon^{(1)}$ & 0.332 & 0.564 & 0.383 & 0.535 & 0.343 & 0.557 & 1.487 & 0.222 & 2.383 & 0.122 \\
\hline$\Upsilon^{(2)}$ & 0.198 & 0.655 & 0.244 & 0.621 & 0.113 & 0.735 & 0.260 & 0.609 & 0.045 & 0.830 \\
\hline$\Upsilon^{(3)}$ & 2.394 & 0.121 & 2.446 & 0.117 & 2.400 & 0.121 & 3.263 & 0.070 & 3.124 & 0.077 \\
\hline$\Upsilon^{(4)}$ & 0.337 & 0.560 & 0.550 & 0.458 & 0.556 & 0.455 & 4.910 & 0.026 & 4.932 & 0.026 \\
\hline$\Upsilon^{(5)}$ & 0.024 & 0.875 & 0.034 & 0.853 & 0.072 & 0.787 & 0.032 & 0.855 & 0.050 & 0.822 \\
\hline
\end{tabular}

The tests on serial correlation in the SACPH residuals indicate that the null hypothesis of no serial correlation cannot be rejected in nearly all cases. Thus, the SACPH model does a good job in capturing the dynamics in the data. In order to examine the influence of the chosen categorization and the number of the categories on the estimates of the dynamic parameters $\hat{\alpha}_{1}$ and $\hat{\beta}_{1}$, we re-estimate the individual models based on different groupings of the left hand variable. Hence, while specification (1) corresponds to a highly parameterized model leading to a quite precise estimation of the underlying baseline survivor function, specification (5) is based on only six categories associated with boundaries of 24, 72, 120, 168 and 240 hours. We observe that the estimates of $\alpha_{1}$ and $\beta_{1}$ remain remarkably stable over all particular regressions. Nevertheless, we observe changes in the estimates of the corresponding threshold parameters, as well as of the heterogeneity parameter $\eta$. These differences are due to the fact that the distributional properties of the generalized error $e_{i}$ change whenever we use a different categorization. 
A useful check of the results' robustness over the particular specifications is to compare the resulting estimates of the corresponding discrete points of the baseline distribution function. Table 6 shows the estimated conditional failure probabilities associated with the corresponding category boundaries ${ }^{20}$. Again, we observe quite stable estimates over the individual specifications which confirms our findings from the Monte Carlo study. These results illustrate that the SACPH model works nicely even based on a very small number of categories. Hence, the model dynamics can be estimated quite precisely while ensuring model parsimony. Thus, by applying the SACPH model, it is sufficient to estimate only those points of the conditional duration distribution function which are of particular interest in the corresponding application.

Table 5: ML estimates of $\mathrm{SACPH}(1,1)$ models for Bund future price durations using 50 tick price changes. Standard errors computed based on robust estimates of the covariance matrix. Diagnostics: Log Likelihood (LL), as well as test statistics for a test on serial correlation (inclusive p-values) of the $\mathrm{SACPH}$ residuals.

\begin{tabular}{|c|c|c|c|c|c|c|c|c|c|c|}
\hline & \multicolumn{2}{|c|}{ (1) } & \multicolumn{2}{|c|}{$(2)$} & \multicolumn{2}{|c|}{ (3) } & \multicolumn{2}{|c|}{ (4) } & \multicolumn{2}{|c|}{ (5) } \\
\hline & est. & S.E. & est. & S.E. & est. & S.E. & est. & S.E. & est. & S.E. \\
\hline \multicolumn{11}{|c|}{ Thresholds } \\
\hline$\nu_{1}^{*}\left(\bar{x}_{1}=1 h\right)$ & -3.321 & 1.290 & -3.304 & 0.61 & & & & & & \\
\hline$\nu_{2}^{*}\left(\bar{x}_{2}=2 h\right)$ & -1.779 & 1.243 & -1.762 & 0.55 & & & & & & \\
\hline$\nu_{3}^{*}\left(\bar{x}_{3}=3 h\right)$ & -1.247 & 1.247 & -1.230 & 0.55 & & & & & & \\
\hline$\nu_{4}^{*}\left(\bar{x}_{4}=4 h\right)$ & -1.002 & 1.251 & -0.985 & 0.55 & -1.146 & 1.252 & & & & \\
\hline$\nu_{5}^{*}\left(\bar{x}_{5}=5 h\right)$ & -0.804 & 1.241 & -0.787 & 0.56 & & & & & & \\
\hline$\nu_{6}^{*}\left(\bar{x}_{6}=6 h\right)$ & -0.596 & 1.248 & -0.578 & 0.56 & & & & & & \\
\hline$\nu_{7}^{*}\left(\bar{x}_{7}=7 h\right)$ & -0.261 & 1.259 & -0.243 & 0.57 & & & & & & \\
\hline$\nu_{8}^{*}\left(\bar{x}_{8}=8 h\right)$ & -0.073 & 1.269 & -0.056 & 0.57 & -0.235 & 1.270 & & & & \\
\hline$\nu_{9}^{*}\left(\bar{x}_{9}=9 h\right)$ & 0.091 & 1.278 & 0.108 & 0.57 & & & & & & \\
\hline$\nu_{10}^{*}\left(\bar{x}_{10}=24 h\right)$ & 0.752 & 1.317 & 0.769 & 0.58 & 0.567 & 1.315 & 1.268 & 1.177 & 1.301 & 1.175 \\
\hline$\nu_{11}^{*}\left(\bar{x}_{11}=30 h\right)$ & 1.177 & 1.342 & & & & & & & & \\
\hline$\nu_{11}^{*}\left(\bar{x}_{11}=36 h\right)$ & 1.316 & 1.356 & 1.344 & 0.59 & 1.135 & 1.351 & & & & \\
\hline$\nu_{12}^{*}\left(\bar{x}_{12}=48 h\right)$ & 1.647 & 1.386 & 1.672 & 0.60 & 1.459 & 1.377 & 2.176 & 1.233 & & \\
\hline$\nu_{13}^{*}\left(\bar{x}_{13}=72 h\right)$ & 2.200 & 1.431 & 2.223 & 0.62 & 2.002 & 1.418 & 2.727 & 1.267 & 2.779 & 1.268 \\
\hline$\nu_{14}^{*}\left(\bar{x}_{14}=96 h\right)$ & 2.580 & 1.468 & 2.602 & 0.63 & 2.375 & 1.451 & 3.107 & 1.293 & & \\
\hline$\nu_{15}^{*}\left(\bar{x}_{15}=120 h\right)$ & 3.039 & 1.515 & 3.060 & 0.65 & 2.825 & 1.494 & 3.566 & 1.327 & 3.627 & 1.330 \\
\hline$\nu_{16}^{*}\left(\bar{x}_{16}=144 h\right)$ & 3.435 & 1.563 & 3.455 & 0.67 & 3.212 & 1.535 & 3.960 & 1.362 & & \\
\hline$\nu_{17}^{*}\left(\bar{x}_{17}=168 h\right)$ & 4.010 & 1.643 & 4.028 & 0.70 & 3.773 & 1.607 & 4.531 & 1.424 & 4.596 & 1.429 \\
\hline$\nu_{18}^{*}\left(\bar{x}_{18}=192 h\right)$ & 4.434 & 1.703 & 4.449 & 0.73 & 4.182 & 1.661 & 4.950 & 1.472 & & \\
\hline$\nu_{19}^{*}\left(\bar{x}_{19}=240 h\right)$ & 4.963 & 1.795 & 4.974 & 0.77 & 4.691 & 1.741 & 5.469 & 1.538 & 5.561 & 1.551 \\
\hline$\nu_{19}^{*}\left(\bar{x}_{19}=288 h\right)$ & 5.493 & 1.882 & & & & & & & & \\
\hline$\alpha_{1}$ & 0.975 & 0.013 & 0.975 & 0.01 & 0.977 & 0.012 & 0.982 & 0.011 & 0.982 & 0.011 \\
\hline$\beta_{1}$ & -0.872 & 0.030 & -0.872 & 0.02 & -0.874 & 0.029 & -0.876 & 0.030 & -0.874 & 0.030 \\
\hline$\eta$ & 0.836 & 0.176 & 0.838 & 0.08 & 0.867 & 0.171 & 0.833 & 0.144 & 0.828 & 0.146 \\
\hline Obs & \multicolumn{2}{|c|}{484} & \multicolumn{2}{|c|}{484} & \multicolumn{2}{|c|}{484} & \multicolumn{2}{|c|}{484} & \multicolumn{2}{|c|}{484} \\
\hline LL & \multicolumn{2}{|c|}{-1249} & \multicolumn{2}{|c|}{-1207} & \multicolumn{2}{|c|}{-1040} & \multicolumn{2}{|c|}{-811} & \multicolumn{2}{|c|}{-647} \\
\hline & est. & p-val. & est. & p-val. & est. & p-val. & est. & p-val. & est. & p-val. \\
\hline$\Upsilon^{(1)}$ & 2.953 & 0.085 & 2.882 & 0.089 & 1.904 & 0.167 & 2.259 & 0.132 & 2.093 & 0.147 \\
\hline$\Upsilon^{(2)}$ & 1.758 & 0.184 & 0.950 & 0.329 & 1.475 & 0.224 & 2.967 & 0.084 & 3.505 & 0.061 \\
\hline$\Upsilon^{(3)}$ & 1.636 & 0.200 & 0.863 & 0.352 & 0.667 & 0.414 & 0.107 & 0.743 & 0.246 & 0.619 \\
\hline$\Upsilon^{(4)}$ & 1.100 & 0.294 & 0.773 & 0.379 & 1.573 & 0.209 & 2.910 & 0.088 & 2.850 & 0.091 \\
\hline$\Upsilon^{(5)}$ & 2.069 & 0.150 & 1.932 & 0.164 & 2.180 & 0.139 & 1.891 & 0.169 & 2.016 & 0.155 \\
\hline
\end{tabular}

\footnotetext{
${ }^{20}$ Here, $e_{i}$ and $\phi_{i}$ are set to their unconditional means.
} 
Table 6: Failure probabilities based on Bund future price durations using 30, 40 and 50 tick price changes. Based on the estimates in Tables 3, 4 and 5 .

\begin{tabular}{|c|c|c|c|c|c|c|c|c|c|c|}
\hline & \multicolumn{5}{|c|}{30 ticks } & \multicolumn{5}{|c|}{40 ticks } \\
\hline & (1) & $(2)$ & $(3)$ & (4) & $(5)$ & (1) & $(2)$ & $(3)$ & $(4)$ & $(5)$ \\
\hline $\operatorname{Pr}\left[x_{i}<=0.5 h\right]$ & 0.032 & & & & & 0.014 & & & & \\
\hline $\operatorname{Pr}\left[x_{i}<=1 h\right]$ & 0.080 & 0.079 & & & & 0.037 & 0.037 & & & \\
\hline $\operatorname{Pr}\left[x_{i}<=2 h\right]$ & 0.166 & 0.164 & & & & 0.083 & 0.083 & & & \\
\hline $\operatorname{Pr}\left[x_{i}<=3 h\right]$ & 0.230 & 0.227 & & & & 0.126 & 0.126 & & & \\
\hline $\operatorname{Pr}\left[x_{i}<=4 h\right]$ & 0.286 & 0.283 & 0.294 & & & 0.153 & 0.153 & 0.156 & & \\
\hline $\operatorname{Pr}\left[x_{i}<=5 h\right]$ & 0.326 & 0.323 & & & & 0.186 & 0.186 & & & \\
\hline $\operatorname{Pr}\left[x_{i}<=6 h\right]$ & 0.378 & 0.374 & & & & 0.218 & 0.217 & & & \\
\hline $\operatorname{Pr}\left[x_{i}<=7 h\right]$ & 0.422 & 0.418 & & & & 0.262 & 0.261 & & & \\
\hline $\operatorname{Pr}\left[x_{i}<=8 h\right]$ & 0.457 & 0.452 & 0.463 & & & 0.296 & 0.294 & 0.300 & & \\
\hline $\operatorname{Pr}\left[x_{i}<=9 h\right]$ & 0.480 & 0.476 & & & & 0.311 & 0.309 & & & \\
\hline $\operatorname{Pr}\left[x_{i}<=24 h\right]$ & 0.714 & 0.710 & 0.715 & 0.723 & 0.724 & 0.496 & 0.494 & 0.496 & 0.498 & 0.496 \\
\hline $\operatorname{Pr}\left[x_{i}<=28 h\right]$ & 0.771 & & & & & 0.580 & & & & \\
\hline $\operatorname{Pr}\left[x_{i}<=32 h\right]$ & 0.808 & & & & & 0.627 & & & & \\
\hline $\operatorname{Pr}\left[x_{i}<=36 h\right]$ & 0.810 & 0.811 & 0.814 & & & 0.629 & 0.634 & 0.635 & & \\
\hline $\operatorname{Pr}\left[x_{i}<=48 h\right]$ & 0.836 & 0.836 & 0.838 & 0.843 & & 0.677 & 0.680 & 0.681 & 0.682 & \\
\hline $\operatorname{Pr}\left[x_{i}<=72 h\right]$ & 0.897 & 0.896 & 0.898 & 0.900 & 0.901 & 0.795 & 0.794 & 0.795 & 0.795 & 0.796 \\
\hline $\operatorname{Pr}\left[x_{i}<=96 h\right]$ & 0.943 & 0.943 & 0.943 & 0.944 & & 0.857 & 0.856 & 0.857 & 0.857 & \\
\hline $\operatorname{Pr}\left[x_{i}<=120 h\right]$ & 0.968 & 0.968 & 0.968 & 0.968 & 0.969 & 0.913 & 0.913 & 0.913 & 0.913 & 0.915 \\
\hline $\operatorname{Pr}\left[x_{i}<=144 h\right]$ & 0.985 & 0.985 & 0.985 & 0.985 & & 0.928 & 0.928 & 0.929 & 0.929 & \\
\hline $\operatorname{Pr}\left[x_{i}<=168 h\right]$ & 0.990 & 0.990 & 0.990 & 0.990 & 0.990 & 0.956 & 0.956 & 0.956 & 0.956 & 0.957 \\
\hline $\operatorname{Pr}\left[x_{i}<=192 h\right]$ & 0.995 & 0.996 & 0.996 & 0.996 & & 0.968 & 0.968 & 0.968 & 0.968 & \\
\hline $\operatorname{Pr}\left[x_{i}<=240 h\right]$ & 0.996 & 0.997 & 0.997 & 0.997 & 0.997 & 0.977 & 0.978 & 0.978 & 0.977 & 0.978 \\
\hline \multicolumn{6}{|c|}{50 ticks } & & & & & \\
\hline & (1) & $(2)$ & $(3)$ & (4) & $(5)$ & & & & & \\
\hline $\operatorname{Pr}\left[x_{i}<=1 h\right]$ & 0.009 & 0.009 & & & & & & & & \\
\hline $\operatorname{Pr}\left[x_{i}<=2 h\right]$ & 0.041 & 0.041 & & & & & & & & \\
\hline $\operatorname{Pr}\left[x_{i}<=3 h\right]$ & 0.068 & 0.068 & & & & & & & & \\
\hline $\operatorname{Pr}\left[x_{i}<=4 h\right]$ & 0.085 & 0.085 & 0.091 & & & & & & & \\
\hline $\operatorname{Pr}\left[x_{i}<=5 h\right]$ & 0.101 & 0.102 & & & & & & & & \\
\hline $\operatorname{Pr}\left[x_{i}<=6 h\right]$ & 0.122 & 0.122 & & & & & & & & \\
\hline $\operatorname{Pr}\left[x_{i}<=7 h\right]$ & 0.162 & 0.162 & & & & & & & & \\
\hline $\operatorname{Pr}\left[x_{i}<=8 h\right]$ & 0.189 & 0.189 & 0.197 & & & & & & & \\
\hline $\operatorname{Pr}\left[x_{i}<=9 h\right]$ & 0.215 & 0.215 & & & & & & & & \\
\hline $\operatorname{Pr}\left[x_{i}<=24 h\right]$ & 0.342 & 0.342 & 0.350 & 0.345 & 0.342 & & & & & \\
\hline $\operatorname{Pr}\left[x_{i}<=30 h\right]$ & 0.439 & & & & & & & & & \\
\hline $\operatorname{Pr}\left[x_{i}<=36 h\right]$ & 0.471 & 0.474 & 0.481 & & & & & & & \\
\hline $\operatorname{Pr}\left[x_{i}<=48 h\right]$ & 0.549 & 0.551 & 0.558 & 0.554 & & & & & & \\
\hline $\operatorname{Pr}\left[x_{i}<=72 h\right]$ & 0.670 & 0.672 & 0.678 & 0.674 & 0.675 & & & & & \\
\hline $\operatorname{Pr}\left[x_{i}<=96 h\right]$ & 0.742 & 0.743 & 0.749 & 0.745 & & & & & & \\
\hline $\operatorname{Pr}\left[x_{i}<=120 h\right]$ & 0.812 & 0.813 & 0.818 & 0.815 & 0.816 & & & & & \\
\hline $\operatorname{Pr}\left[x_{i}<=144 h\right]$ & 0.860 & 0.861 & 0.865 & 0.862 & & & & & & \\
\hline $\operatorname{Pr}\left[x_{i}<=168 h\right]$ & 0.910 & 0.911 & 0.914 & 0.911 & 0.912 & & & & & \\
\hline $\operatorname{Pr}\left[x_{i}<=192 h\right]$ & 0.936 & 0.936 & 0.938 & 0.936 & & & & & & \\
\hline $\operatorname{Pr}\left[x_{i}<=240 h\right]$ & 0.958 & 0.958 & 0.960 & 0.958 & 0.959 & & & & & \\
\hline $\operatorname{Pr}\left[x_{i}<=288 h\right]$ & 0.973 & & & & & & & & & \\
\hline
\end{tabular}

7.2. A Forecasting Evaluation. In order to compare the performance of the SACPH model to that of alternative dynamic duration models, we evaluate the competing models based on their in-sample and out-of-sample forecasting power. As suggested by Dufour and Engle (2000) and Bauwens, Giot, Grammig, and Veredas (2004) a natural and powerful way to assess a model's forecasting performance is to evaluate the model on the basis of density forecasts. The advantage of this procedure is that it is easily implemented and that it allows to compare nonnested models regardless of the user's loss function. Moreover, evaluating the complete forecast density (instead of just the first moment) provides a more thorough assessment of the model. 
Denote $\left\{p_{i}\left(x_{i} \mid \mathcal{F}_{i-1}\right)\right\}_{i=1}^{n}$ as the sequence of densities associated with the underlying DGP of $x_{i}$. Correspondingly, $\left\{f_{i}\left(x_{i} \mid \mathcal{F}_{i-1}\right)\right\}_{i=1}^{n}$ defines the sequence of one-step-ahead density forecasts produced by the model. Diebold, Gunther, and Tay (1998) propose to test the null hypothesis $\left\{f_{i}\left(x_{i} \mid \mathcal{F}_{i-1}\right)\right\}_{i=1}^{n}=\left\{p_{i}\left(x_{i} \mid \mathcal{F}_{i-1}\right)\right\}_{i=1}^{n}$ by evaluating the probability integral transform (PIT)

$$
\xi_{i}:=\int_{-\infty}^{x_{i}} f_{i}(s) d s
$$

Building on the results by Rosenblatt (1952), Diebold, Gunther, and Tay (1998) show that under the null hypothesis, the $\xi_{i}$ 's are i.i.d. uniformly distributed.

A difficulty in the SACPH framework is that the durations are categorized and thresholds $\mu_{k}^{*}$ are only estimated for the corresponding category bounds. A simple possibility to overcome this problem, is to linearly interpolate (and extrapolate, respectively) the estimated threshold parameters $\mu_{k}^{*}, k=1, \ldots, K-1$ over the range of the observed durations. Then, by denoting the interpolated thresholds associated with a realization $x_{i}$ by $\breve{\mu}_{i}$, the corresponding PIT (for a non-censored observation) is straightforwardly computed as

$$
\xi_{i}=F_{\epsilon^{*}}\left(\breve{\mu}_{i}-\phi_{i}\right) \quad \text { if } x_{i} \text { is non-censored. }
$$

The consideration of censored observations is more difficult since they are per definition not observable. The most appropriate solution is to evaluate the PIT of a censored observation at the value of its conditional expectation given the lower and upper category bound. This is easily performed by computing the PIT for a censored observation based on the corresponding generalized residual, i.e.

$$
\xi_{i}=F_{\epsilon^{*}}\left(e_{i}\right) \quad \text { if } x_{i} \text { is censored. }
$$

In order to assess the forecasting performance of the SACPH model we suggest to compare it to that of an ACD model which is by far the most prominent dynamic duration model in the literature of financial point processes and whose performance has been already extensively analyzed in several evaluation studies, such as in Bauwens, Giot, Grammig, and Veredas (2004), Meitz and Teräsvirta (2006) or Hautsch (2006). In the ACD model, durations $x_{i}$ follow a multiplicative error structure of the form

$$
x_{i}=\Psi_{i} \varepsilon_{i}
$$

where $\Psi_{i}:=\mathrm{E}\left[x_{i} \mid \mathcal{F}_{i-1}\right]$ denotes the conditional expected duration and $\varepsilon_{i}$ is positive valued i.i.d. error term with $\mathrm{E}\left[\varepsilon_{i}\right]=1$. Engle and Russell (1998) propose to specify $\Psi_{i}$ in terms of an autoregressive process of the form

$$
\Psi_{i}=\omega+\sum_{j=1}^{p} \alpha_{j} x_{i-j}+\sum_{j=1}^{q} \beta_{j} \Psi_{i-j}
$$

leading to a linear $\operatorname{ACD}(p, q)$ model. It can be easily shown that the $\operatorname{ACD}$ model belongs to the class of accelerated failure time (AFT) models (see e.g. Engle and Russell, 1998) in the sense that $\Psi_{i}$ accelerates or decelerates the time to failure. One of the most prominent variations is 
the Log-ACD model proposed by Bauwens and Giot (2000) which does not require any nonnegativity constraints and is given by

$$
\ln \Psi_{i}=\omega+\sum_{j=1}^{p} \alpha_{j} \ln x_{i-j}+\sum_{j=1}^{q} \beta_{j} \ln \Psi_{i-j} .
$$

Table 7: Evaluation of one-step-ahead in-sample density forecasts based on Bund future price durations using 30,40 and 50 tick price changes. The density forecasts are computed using the SACPH estimates shown in Tables 3, 4 and 5 and using the estimates of (Log-)ACD models based on a generalized gamma and Burr distribution, respectively. The table shows Ljung-Box statistics and $\chi^{2}$-goodness-of-fit test statistics based on the $\xi_{i}$ series as well as the corresponding p-values. The first two test statistics are computed based on the complete $\xi_{i}$ series. Here, censored observations are evaluated at their observable minimum. The test statistics indexed by a ${ }^{, *}$, are computed based on realizations of $\xi_{i}$ which are not subjected to censoring.

\begin{tabular}{|c|c|c|c|c|c|c|c|c|c|}
\hline \multicolumn{10}{|c|}{30 ticks } \\
\hline & SACPH(1) & SACPH(2) & SACPH(3) & SACPH(4) & SACPH(5) & GG-ACD & B-ACD & GG-LACD & B-LACD \\
\hline$\chi_{19}^{2}$ & 20.003 & 39.938 & 181.11 & 493.90 & 523.98 & 89.400 & 72.499 & 65.713 & 74.489 \\
\hline p-val & 0.394 & 0.003 & 0.000 & 0.000 & 0.000 & 0.000 & 0.000 & 0.000 & 0.000 \\
\hline $\mathrm{LB}(20)$ & 24.606 & 22.461 & 21.449 & 1046.9 & 1127.8 & 33.943 & 28.397 & 21.169 & 26.725 \\
\hline p-val & 0.216 & 0.316 & 0.371 & 0.000 & 0.000 & 0.027 & 0.100 & 0.387 & 0.143 \\
\hline$\chi_{19}^{2 *}$ & 37.346 & 56.538 & 122.536 & 630.80 & 677.00 & 61.308 & 59.077 & 69.654 & 79.962 \\
\hline p-val ${ }^{*}$ & 007 & .000 & 0.000 & 0.000 & 0.000 & 0.000 & 0.000 & 0.000 & 0.000 \\
\hline $\mathrm{LB}(2$ & 16.011 & 16.175 & 25.227 & 2418.0 & 2705.3 & 31.036 & 21.752 & 19.409 & 15.978 \\
\hline $\mathrm{p}$-val ${ }^{*}$ & 715 & 0.705 & 0.192 & 0.000 & 0.000 & 0.055 & 0.354 & 0.495 & 0.718 \\
\hline \multicolumn{10}{|c|}{40 ticks } \\
\hline & SACPH(1) & $\mathrm{SACPH}(2)$ & SACPH(3) & SACPH(4) & $\overline{\mathrm{SACPH}(5)}$ & GG-ACD & B-ACD & GG-LACD & $\overline{B-L A C D}$ \\
\hline$\chi_{19}^{2}$ & 17.194 & 19.140 & 47.523 & 121.85 & 132.84 & 49.298 & 53.303 & 61.830 & 60.685 \\
\hline p-val & 576 & 447 & 000 & 000 & 000 & 000 & 0.000 & 0.000 & 0.000 \\
\hline $\mathrm{LB}(20)$ & 12.450 & 13.017 & 13.542 & 30.450 & 34.305 & 18.436 & 18.049 & 17.311 & 17.402 \\
\hline p-val & 899 & 0.876 & 0.852 & 0.062 & 0.024 & 0.559 & 0.584 & 0.633 & 0.627 \\
\hline$\chi_{19}^{2 *}$ & 28.068 & 28.137 & 46.206 & 165.726 & 178.75 & 50.759 & 39.862 & 58.000 & 57.448 \\
\hline al ${ }^{*}$ & 0.082 & 30 & 0.000 & 0.000 & 0.000 & 0.000 & 0.003 & 0.000 & 0.000 \\
\hline $\operatorname{LB}(20)^{*}$ & 16.338 & 15.940 & 17.329 & 51.498 & 58.882 & 18.036 & 17.330 & 17.672 & 17.464 \\
\hline p-val ${ }^{*}$ & 0.695 & 0.720 & 0.631 & 0.000 & 0.000 & 0.585 & 0.631 & 0.609 & 0.623 \\
\hline \multicolumn{10}{|c|}{50 ticks } \\
\hline & SACPH(1) & $\mathrm{SACPH}(2)$ & $\mathrm{SACPH}(3)$ & $\mathrm{SACPH}(4)$ & SACPH(5) & GG-ACD & B-ACD & GG-LACD & B-LACD \\
\hline$\chi_{19}^{2}$ & 16.578 & 19.636 & 45.586 & 52.942 & 54.595 & 29.719 & 35.917 & 37.570 & 35.917 \\
\hline & 0.618 & 0.416 & 0.000 & 0.000 & 0.000 & 0.055 & 0.011 & 0.007 & 0.011 \\
\hline $\mathrm{LB}(20)$ & 27.541 & 27.405 & 30.014 & 37.679 & 40.587 & 49.488 & 46.580 & 49.393 & 46.580 \\
\hline p-val & 0.120 & 0.124 & 0.069 & 0.009 & 0.004 & 0.000 & 0.001 & 0.000 & 0.001 \\
\hline$\chi_{19}^{2 *}$ & 21.265 & 23.874 & 37.980 & 65.323 & 68.222 & 23.295 & 30.541 & 38.367 & 30.541 \\
\hline & 22 & 0.201 & 0.005 & 0.000 & 0.000 & 0.225 & 0.045 & 0.005 & 0.045 \\
\hline $\operatorname{LB}(20)^{*}$ & 12.105 & 12.551 & 12.760 & 15.175 & 18.990 & 28.936 & 29.679 & 32.324 & 29.679 \\
\hline p-val ${ }^{*}$ & 0.912 & 0.895 & 0.887 & 0.766 & 0.522 & 0.089 & 0.075 & 0.040 & 0.075 \\
\hline
\end{tabular}

A simple choice for the distribution of the ACD error $\varepsilon_{i}$ is an exponential distribution which however is clearly too restrictive in most applications. Formulating an ACD model based on generalized error term structures, such as e.g. the generalized gamma distribution based on the parameters $\phi_{i}, a$ and $m$ (see Lunde, 2000), leads to

$$
\Psi_{i}=\phi_{i} \zeta(a, m)
$$

where

$$
\zeta(a, m):=\frac{\Gamma(m+1 / a)}{\Gamma(m)},
$$

$\Gamma(\cdot)$ denotes the gamma function, and the time-varying scaling parameter $\phi_{i}$ captures the serial dependence of the process. Then, assuming for simplicity a linear $\operatorname{ACD}(1,1)$ specification, $\phi_{i}$ is 
given by

$$
\phi=\frac{\omega}{\zeta(a, m, \eta)}+\frac{\alpha}{\zeta(a, m, \eta)} x_{i-1}+\beta \phi_{i-1}
$$

leading the $\log$ likelihood function

$$
\ln \mathcal{L}(W ; \theta)=\sum_{i=1}^{n} \ln (a)-\ln \Gamma(m)-a m \ln \phi_{i}+(a m-1) \ln x_{i}-\left(x_{i} / \phi_{i}\right)^{a} .
$$

ACD specifications based on alternative distributions, like the generalized F distribution (Hautsch, 2003) or the Burr distribution (Grammig and Maurer, 2000), are obtained similarly.

Table 8: Evaluation of one-step-ahead out-of-sample density forecasts based on Bund future price durations using 30, 40 and 50 tick price changes. The density forecasts are evaluated based on the last $25 \%$ of the sample using SACPH and (Log-) ACD estimates which are produced based on the first $75 \%$ of the sample. The SACPH estimates are based on categorizations as shown in the Tables 3,4 and 5. The ACD estimates are based on estimates of (Log-)ACD models with a generalized gamma and Burr distribution, respectively. The table shows Ljung-Box statistics and $\chi^{2}$-goodness-of-fit test statistics based on the $\xi_{i}$ series as well as the corresponding p-values. The first two test statistics are computed based on the complete $\xi_{i}$ series. Here, censored observations are evaluated at their observable minimum. The test statistics indexed by a ${ }^{*}$, are computed based on realizations of $\xi_{i}$

\begin{tabular}{|c|c|c|c|c|c|c|c|c|c|}
\hline \multicolumn{10}{|c|}{30 ticks } \\
\hline & SACPH(1) & SACPH(2) & SACPH(3) & $\mathrm{SACPH}(4)$ & $\mathrm{SACPH}(5)$ & GG-ACD & B-ACD & GG-LACD & B-LACD \\
\hline$\chi_{19}^{2}$ & 37.820 & 36.127 & 59.188 & 137.23 & 141.27 & 23.228 & 29.091 & 17.495 & 30.915 \\
\hline p-val & 0.006 & 0.010 & 0.000 & 0.000 & 0.000 & 0.227 & 0.065 & 0.556 & 0.041 \\
\hline $\mathrm{LB}(20)$ & 21.232 & 20.791 & 23.271 & 206.06 & 207.08 & 16.381 & 17.873 & 21.073 & 25.849 \\
\hline p-val & 0.383 & 0.409 & 0.275 & 0.000 & 0.000 & 0.693 & 0.596 & 0.393 & 0.171 \\
\hline$\chi_{19}^{2 *}$ & 41.624 & 36.090 & 40.201 & 145.65 & 161.78 & 19.016 & 24.075 & 27.395 & 21.229 \\
\hline p-val* & 0.001 & 0.010 & 0.003 & 0.000 & 0.000 & 0.456 & 0.193 & 0.096 & 0.324 \\
\hline $\mathrm{LB}(20)^{*}$ & 18.980 & 19.387 & 22.722 & 304.04 & 337.79 & 21.590 & 20.393 & 19.763 & 20.736 \\
\hline p-val* & 0.523 & 0.496 & 0.302 & 0.000 & 0.000 & 0.363 & 0.434 & 0.473 & 0.413 \\
\hline \multicolumn{10}{|c|}{40 ticks } \\
\hline & $\mathrm{SACPH}(1)$ & $\mathrm{SACPH}(2)$ & $\mathrm{SACPH}(3)$ & $\mathrm{SACPH}(4)$ & $\mathrm{SACPH}(5)$ & GG-ACD & B-ACD & GG-LACD & B-LACD \\
\hline$\chi_{19}^{2}$ & 31.742 & 24.657 & 30.142 & 30.142 & 29.000 & 21.686 & 23.971 & 32.429 & 31.743 \\
\hline p-val & 0.033 & 0.172 & 0.050 & 0.050 & 0.065 & 0.300 & 0.197 & 0.028 & 0.033 \\
\hline $\mathrm{LB}(20)$ & 17.623 & 17.519 & 16.794 & 14.374 & 15.145 & 14.736 & 14.740 & 16.831 & 16.853 \\
\hline p-val & 0.612 & 0.619 & 0.666 & 0.810 & 0.767 & 0.791 & 0.791 & 0.664 & 0.662 \\
\hline$\chi_{19}^{2 *}$ & 27.507 & 24.318 & 29.536 & 33.594 & 29.826 & 19.391 & 20.841 & 26.348 & 27.507 \\
\hline p-val* & 0.093 & 0.184 & 0.058 & 0.020 & 0.054 & 0.432 & 0.346 & 0.121 & 0.093 \\
\hline $\mathrm{LB}(20)^{*}$ & 12.853 & 13.178 & 14.722 & 14.551 & 15.278 & 11.769 & 11.634 & 12.191 & 12.130 \\
\hline p-val* & 0.883 & 0.869 & 0.792 & 0.801 & 0.760 & 0.924 & 0.928 & 0.909 & 0.912 \\
\hline \multicolumn{10}{|c|}{50 ticks } \\
\hline & $\mathrm{SACPH}(1)$ & $\mathrm{SACPH}(2)$ & $\mathrm{SACPH}(3)$ & $\mathrm{SACPH}(4)$ & SACPH(5) & GG-ACD & B-ACD & GG-LACD & B-LACD \\
\hline$\chi_{19}^{2}$ & 25.942 & 32.553 & 27.264 & 30.239 & 32.884 & 14.372 & 13.380 & 14.702 & 13.380 \\
\hline p-val & 0.131 & 0.027 & 0.098 & 0.048 & 0.024 & 0.762 & 0.819 & 0.741 & 0.819 \\
\hline $\mathrm{LB}(20)$ & 25.671 & 24.317 & 24.728 & 30.118 & 30.784 & 27.064 & 23.826 & 26.012 & 23.826 \\
\hline p-val & 0.176 & 0.228 & 0.212 & 0.067 & 0.058 & 0.133 & 0.250 & 0.165 & 0.250 \\
\hline$\chi_{19}^{2 *}$ & 30.156 & 34.862 & 29.372 & 33.686 & 32.509 & 14.078 & 13.294 & 16.039 & 13.294 \\
\hline p-val* & 0.049 & 0.014 & 0.060 & 0.020 & 0.027 & 0.779 & 0.823 & 0.655 & 0.823 \\
\hline $\mathrm{LB}(20)^{*}$ & 16.347 & 16.305 & 16.970 & 18.191 & 17.478 & 17.881 & 17.842 & 18.380 & 17.842 \\
\hline p-val* & 0.694 & 0.697 & 0.654 & 0.574 & 0.621 & 0.595 & 0.598 & 0.562 & 0.598 \\
\hline
\end{tabular}
which are not subjected to censoring.

As an useful benchmark for the SACPH model we estimate $\operatorname{ACD}(1,1)$ and $\log -\operatorname{ACD}(1,1)$ models with generalized gamma as well as Burr distributed error terms. ${ }^{21}$ As argued above, a main

\footnotetext{
${ }^{21}$ We also estimate ACD models based on the generalized $\mathrm{F}$ distribution. However, in nearly all cases it turns out that the additional flexibility provided by the generalized F distribution is not supported by the data.
} 
drawback of ACD-type models is that censoring is not easily taken into account since the autoregressive structure has to be updated by past realizations of the observed duration process. Since a more-in-depth treatment of this issue is clearly beyond the scope of this paper, we choose a more pragmatic solution based on two alternative estimation strategies. Firstly, we estimate the ACD models using the complete series $x_{i}$ and evaluate censored observations at their corresponding lower limit (as defined in see Section 5.1). Alternatively, we simply skip all censored observations and estimate and evaluate the model based on non-censored durations only. ${ }^{22}$ Then, in both strategies the PIT's are straightforwardly computed on the basis of the implied one-step-ahead forecasted densities using (38).

The competing density forecasts are evaluated by testing the distributional and dynamical properties of the resulting $\xi_{i}$ series. The latter are checked based on Ljung-Box statistics (with respect to 20 lags). The former are tested based on a test against the uniform distribution on the basis of a simple $\chi^{2}$-goodness-of-fit test of the form

$$
\chi^{2}=\sum_{k=1}^{K} \frac{\left(n_{k}-n / K\right)^{2}}{n / K} \sim \chi_{K-1}^{2}
$$

where $K$ denotes the number of categories dividing the range $[0 ; 1]$ in equal categories and $n_{k}$ is the number of realizations of $\left\{\xi_{i}\right\}$ lying in category $k$. Table 7 shows the in-sample density forecasting evaluations using $K=20$. Since the ACD model is evaluated based on both the complete series and alternatively only based on non-censored observations, we additionally also report the corresponding SACPH evaluations based on the $\xi_{i}$ series which excludes censored observations. ${ }^{23}$ The results show that the SACPH model on the basis of a sufficient number of categories clearly out-performs the ACD model in terms of distributional and dynamical properties. This picture is robust over all three aggregation levels. Not surprisingly, the forecasting quality of the SACPH model declines when the number of the underlying categories becomes smaller. This is particularly evident for the two quite broad categorizations in specifications (4) and (5), where no categories smaller than $24 \mathrm{~h}$ are used. However, these results clearly indicate that the SACPH model seems to be a reasonable model in order to capture the stochastic properties of the data. Because of its semiparametric nature, it can cover the (conditional) distribution of the durations arbitrarily precise in dependence of the number of chosen categories while simultaneously capturing persistent serial dependencies in the data.

Table 8 shows the corresponding out-of-sample forecasting results. Here, the individual models are estimated using the first $75 \%$ of the sample, whereas one-step-ahead forecasts are produced for the remaining $25 \%$. While the reported Ljung-Box statistics based on the PIT series are qualitatively quite similar for both types of models we see a slightly better performance of

\footnotetext{
${ }^{22}$ In order to facilitate a direct comparison between both competing approaches, we estimate the ACD models on the basis of the raw durations $x_{i}$ without a pre-filtering of seasonality effects. As mentioned above, the latter have not been found to be important on the chosen aggregation level. For sake of brevity, we do not show the ACD estimates in the paper.

${ }^{23}$ These results are indexed by $\mathrm{a}^{*}$.
} 
the ACD type models with respect to the distributional properties of the forecasts. This result is strongest for 30-tick price changes and becomes weaker for higher aggregation levels. For these series it is evident that the qualitative difference in the forecasting power between SACPH models with many categories and those with less categories is significantly smaller than those on the basis of in-sample forecasts. This result clearly illustrates the well-known trade-off between in-sample (over-)fitting and out-of-sample forecasting performance.

Nevertheless, with respect to both the in-sample and out-of-sample performance, we can conclude that the SACPH model seems to be a promising competing model to well-established approaches. We see the strength of the SACPH model particularly in its ability to provide a very satisfying in-sample goodness-of-fit (particularly compared to quite flexible ACD models) while simultaneously producing qualitatively similar out-of-sample forecasts. Moreover, it allows us to rigorously accounting for censoring structures in a dynamic framework (which is not straightforwardly possible in ACD type models). This is particularly important when duration spells are modelled which may last longer than one trading day. Nevertheless, the SACPH model also provides a useful framework for the modelling of high-frequency duration processes. A comparison between SACPH and ACD models based on trade durations or highly frequent price durations is beyond the scope of the current study but is a clear issue for future research.

\section{Conclusions}

In this paper, we have proposed a dynamic extension of a proportional hazard $(\mathrm{PH})$ model in which the baseline hazard is non-specified. The major idea is to use a categorization approach and to exploit the close relationship between PH models for grouped duration data and ordered response models. We reformulated the $\mathrm{PH}$ model as an ordered response model based on extreme value distributed errors with the unobservable log integrated baseline hazard serving as latent variable. In order to account for (persistent) serial dependence in the duration process, we extend the latent equation by an observation driven ARMA dynamic based on generalized errors. In a Monte Carlo study, we illustrated that the proposed dynamic provides a close approximation to the corresponding dynamic based on true errors. Hence, the so-called semiparametric autoregressive conditional proportional hazard (SACPH) model nicely captures the autocorrelation structure in the unobservable log integrated baseline hazard. Furthermore, it was illustrated that the autocorrelation function implied by the estimates of an SACPH model is a close proxy for the autocorrelation function of the observable duration process.

Therefore, the SACPH model has the virtue of being easy to implement, avoiding the computation of cumbersome high-dimensional integrals and being straightforwardly estimated by maximum likelihood. A further advantage is that the estimates of the threshold parameters associated with the individual categories directly provide estimates of discrete points of the unknown baseline survivor function. 
Moreover, we demonstrated that the model allows to account for censoring structures in a dynamic framework and can be easily extended to accommodate unobserved heterogeneity. Diagnostic tests for the SACPH model have been proposed by adopting a test for serial dependence proposed by Gourieroux, Monfort, and Trognon (1985). In an empirical study we employed the SACPH approach to the modelling of financial durations which are subjected to censoring structures. We illustrated the usefulness and flexibility of the approach to estimate the conditional probabilities of observing fundamental (absolute) price changes in a given time interval. It was shown that the model nicely captures the underlying dynamics in the censored data even when only a small number of categories is used. Furthermore, we illustrated how to produce density forecasts and evaluated them on an in-sample and out-of-sample basis against competing (flexible) ACD models. It turned out that the SACPH model provides a significantly better in-sample goodness-of-fit while yielding a widely similar out-of-sample performance.

Overall, we can conclude that a dynamic semiparametric proportional hazard specification provides a reasonable framework to model autoregressive persistent duration series and yields a sensible alternative to AFT type models originating from traditional time series analysis. The major advantage of the proposed model is that it allows us to combine central features of duration data, like censoring or unobserved heterogeneity, with those of persistent autoregressive time series processes. In this sense, the SACPH model fills an important gap in the recent literature on dynamic point processes. Extensions of the proposed framework, like accounting for statedependent baseline hazard functions, non-linear news impact functions in the model dynamics (as heavily discussed in the ACD literature) or multivariate extensions are clear avenues for future research.

\section{APPENDIX}

Testing for Serial Dependence in the SACPH Model. The test on serial dependence is based on the score of the observable model. Along the lines of the work of Gourieroux, Monfort, and Trognon (1985), the observable score is equal to the conditional expectation of the latent score, given the observable categorized variable, i. e.,

$$
\frac{\partial \ln \mathcal{L}(W ; \theta)}{\partial \theta}=\mathrm{E}\left[\frac{\partial \ln \mathcal{L}^{*}\left(L^{*} ; \theta\right)}{\partial \theta} \mid \mathcal{F}_{i}^{d}\right]
$$

where $\ln \mathcal{L}^{*}(\cdot)$ denotes the $\log$ likelihood function of the latent model and $L^{*}$ denotes the $n \times 1$ vector of the realizations of the latent variable, $\ln \Lambda_{0, i}$. Under the assumption of a $\operatorname{BurrII}(\eta)$ distribution for $\epsilon_{i}^{*}$, the $\log$ likelihood function of the latent model is given by

$$
\begin{aligned}
\ln \mathcal{L}^{*}\left(L^{*} ; \theta\right) & =\sum_{i=j+1}^{n} \ln f_{\epsilon^{*}}\left(u_{i}-\alpha_{j} u_{i-j}\right) \\
& =\sum_{i=j+1}^{n}\left[\ln (\eta)+\alpha_{j} u_{i-j}-u_{i}-(\eta+1) \ln \left[1+\exp \left(\alpha_{j} u_{i-j}-u_{i}\right)\right]\right] .
\end{aligned}
$$


Under the null, the score with respect to $\alpha_{j}, s\left(\alpha_{j}\right)$, is given by

$$
\begin{aligned}
s\left(\alpha_{j}\right) & =\mathrm{E}\left[\frac{\partial \ln \mathcal{L}^{*}\left(L^{*} ; \theta\right)}{\partial \alpha_{j}} \mid \mathcal{F}_{i}^{d}\right] \\
& =\sum_{i=j+1}^{n} \mathrm{E}\left[e_{i-j}^{*} \mid \mathcal{F}_{i}^{d}\right]\left[1-(\eta+1) \mathrm{E}\left[\frac{\exp \left(\epsilon_{i}^{*}\right)}{1+\exp \left(\epsilon_{i}^{*}\right)} \mid \mathcal{F}_{t_{i}}^{d}\right]\right] \\
& =\sum_{i=j+1}^{n} e_{i-j}\left[1-(\eta+1) \tilde{e}_{i}\right],
\end{aligned}
$$

where

$$
\tilde{e}_{i}:=\mathrm{E}\left[\frac{\exp \left(\epsilon_{i}^{*}\right)}{1+\exp \left(\epsilon_{i}^{*}\right)} \mid \mathcal{F}_{i}^{d}\right]
$$

Hence,

$$
s\left(\hat{\alpha}_{j}\right)=\sum_{i=j+1}^{n} \hat{e}_{i-j}\left[1-(\hat{\eta}+1) \hat{\tilde{e}}_{i}\right] .
$$

Under the null, the expectation of $\hat{\tilde{e}}_{i}$ is given by $\mathrm{E}\left[\hat{\tilde{e}}_{i}\right]=(\eta+1)^{-1}$, and thus, $\mathrm{E}\left[s\left(\hat{\alpha}_{j}\right)\right]=0$. Exploiting the asymptotic normality of the score (see Gourieroux, Monfort, and Trognon, 1985), i.e.,

$$
\frac{1}{\sqrt{n}} s\left(\alpha_{j}\right) \stackrel{d}{\longrightarrow} N\left(0, \operatorname{plim} \frac{1}{n} \sum_{i=j+1}^{n} e_{i-j}^{2}\left[1-(\eta+1) \tilde{e}_{i}\right]^{2}\right),
$$

a $\chi^{2}$ statistic for the null hypothesis $H_{0}: \alpha_{j}=0$ is obtained by

$$
\Upsilon^{(j)}=\frac{\left[\sum_{i=j+1}^{n} \hat{e}_{i-j}\left[1-(\hat{\eta}+1) \hat{\tilde{e}}_{i}\right]\right]^{2}}{\sum_{i=j+1}^{n} \hat{e}_{i-j}^{2}\left[1-(\hat{\eta}+1) \hat{\tilde{e}}_{i}\right]^{2}} \stackrel{a}{\sim} \chi^{2}(1) .
$$

Correspondingly, for the standard extreme value case, it is straightforwardly shown that

$$
\Upsilon^{(j)}=\frac{\left[\sum_{i=j+1}^{n} \hat{e}_{i-j}\left(\hat{\tilde{e}}_{i}-1\right)\right]^{2}}{\sum_{i=j+1}^{n} \hat{e}_{i-j}^{2}\left[\hat{\tilde{e}}_{i}-1\right]^{2}} \stackrel{a}{\sim} \chi^{2}(1)
$$

with

$$
\tilde{e}_{i}:=\mathrm{E}\left[\exp \left(\epsilon_{i}^{*}\right) \mid \mathcal{F}_{i}^{d}\right]
$$

\section{REFERENCES}

Baumens, L., P. Giot, J. Grammig, And D. Veredas (2004): "A Comparison of Financial Duration Models Via Density Forecasts," International Journal of Forecasting, 20, 589-609.

Bauwens, L., AND P. Giot (2000): "The Logarithmic ACD Model: An Application to the Bid/Ask Quote Process of two NYSE Stocks," Annales d'Economie et de Statistique, 60, 117-149.

Bauwens, L., And N. Hautsch (2006): "Modelling Financial High Frequency Data Using Point Processes," Working Paper University of Copenhagen, Université Catholique de Louvain.

Bauwens, L., And D. Veredas (2004): "The Stochastic Conditional Duration Model: A Latent Factor Model for the Analysis of Financial Durations," Journal of Econometrics, 119, 381-412.

BlazseK, S., AND A. Escribano (2005): "Dynamic Latent Factor Intensity Models of Knowledge Spillovers: Evidence Based on Patent Analysis," Working Paper Universidad Carlos III de Madrid. 
Breslow, N. (1972): "Contribution to the Discussion of the Paper by D. R. Cox," Journal of the Royal Statistical Society, Series B, 34, 216-17.

Cox, D. R., And V. Isham (1980): Point Processes. Chapman and Hall, London.

Cox, D. R., AND D. OAKes (1984): Analysis of Survival Data. Chapman and Hall, London.

Cox, D. R. (1972): "Regression Models and Life Tables," Journal of the Royal Statistical Society, Series B, 34, $187-220$.

(1975): "Partial Likelihood," Biometrika, 62, 269.

Diebold, F. X., T. A. Gunther, And A. S. Tay (1998): "Evaluating Density Forecasts, with Applications to Financial Risk Management," International Economic Review, 39, 863-883.

Dufour, A., And R. F. Engle (2000): "The ACD Model: Predictability of the Time between Consecutive Trades," Working Paper, ISMA Centre, University of Reading.

Engle, R. F., AND J. R. Russell (1998): "Autoregressive Conditional Duration: A New Model for Irregularly Spaced Transaction Data," Econometrica, 66, 1127-1162.

Engle, R. F. (2000): "The Econometrics of Ultra-High-Frequency Data," Econometrica, 68, 1, 1-22.

Fernandes, M., AND J. Grammig (2006): "A Family of Autoregressive Conditional Duration Models," Journal of Econometrics, 130, 1-23.

Gerhard, F., AND N. Hautsch (2002): "Volatility Estimation on the Basis of Price Intensities," Journal of Empirical Finance, 9, 57-89.

Gorgens, T., AND J. L. Horowitz (1999): "Semiparametric Estimation of a Censored Regression Model with an Unknown Transformation of the Dependent Variable," Journal of Econometrics, 90, 155-191.

Gourieroux, C., A. Monfort, E. Renault, And A. Trognon (1987): "Generalised Residuals," Journal of Econometrics, 34, 5-32.

Gourieroux, C., A. Monfort, And A. Trognon (1985): "A General Approach to Serial Correlation," Econometric Theory, pp. 315-340.

Grammig, J., AND K.-O. Maurer (2000): "Non-Monotonic Hazard Functions and the Autoregressive Conditional Duration Model," Econometrics Journal, 3, 16-38.

Gritz, R. M. (1993): "The Impact of Training on the Frequency and Duration of Employment," Journal of Econometrics, 57, 21-51.

Han, A., AND J. A. Hausman (1990): "Flexible Parametric Estimation of Duration and Competing Risk Models," Journal of Applied Econometrics, 5, 1-28.

Hautsch, N. (2003): "Assessing the Risk of Liquidity Suppliers on the Basis of Excess Demand Intensities," Journal of Financial Econometrics, 1, 189-215.

Hautsch, N. (2004): Modelling Irregularly Spaced Financial Data. Springer, Berlin.

(2006): "Testing the Conditional Mean Function of Autoregressive Conditional Duration Models," Working Paper, Department of Economics, University of Copenhagen.

HeCKmann, J. J., AND B. Singer (1984): "Econometrics Duration Analysis," Journal of Econometrics, 24, $63-132$.

Honoré, B. E. (1990): "Simple Estimation of Duration Model with Unobserved Heterogeneity," Econometrica, $58,453-473$.

Horowitz, J. L., And G. R. Neumann (1987): "Semiparametric Estimation of Employment Duration Models," Econometric Review, 6(1), 5-40.

Hujer, R., AND S. Vuletic (2005): "The Regime Switching ACD Framework: The Use of the Comprehensive Family of Distributions," Available at http://ssrn.com/abstract $=766684$.

JAsiAK, J. (1998): "Persistence in Intratrade Durations," Finance, 19, 166-195.

Kalbfleisch, J. D., AND R. L. Prentice (1980): The Statistical Analysis of Failure Time Data. Wiley.

KAPlan, E. L., AND P. Meier (1958): "Nonparametric Estimation from Incomplete Observations," Journal of the American Statistical Association, 53, 457-481.

Kiefer, N. M. (1988): "Economic Duration Data and Hazard Functions," Journal of Economic Literature, 26, 646-679.

Koopman, S. J., A. Lucas, And A. Monteiro (2005): "The Multi-State Latent Factor Intensity Model for Credit Rating Transitions," Discussion Paper TI2005-071/4, Tinbergen Institute.

Lancaster, T. (1979): "Econometric Methods for the Duration of Unemployment," Econometrica, 47 (4), 939956.

(1997): The Econometric Analysis of Transition Data. Cambridge University Press.

Liesenfeld, R., And J.-F. Richard (2003): "Univariate and Multivariate Stochastic Volatility Models: Estimation and Diagnostics," Journal of Empirical Finance, 10, 505-531.

Lunde, A. (2000): "A Generalized Gamma Autoregressive Conditional Duration Model," Discussion paper, University of Aarhus.

MCCALL, B. P. (1996): "Unemployment Insurance Rules, Joblessness and Part-Time Work," Econometrica, 64 (3), 647-682.

Meitz, M., And T. Teräsvirta (2006): "Evaluating Models of Autoregressive Conditional Duration," Journal of Business 83 Economic Statistics, 24, 104-124.

Meyer, B. D. (1990): "Unemployment Insurance and Unemployment Spells," Econometrica, 58 (4), 757-782. 
Moffitt, R. (1985): "Unemployment Insurance and the Distribution of Unemployment Spells," Journal of Econometrics, 28, 85-101.

Neumann, G. (1997): Search Models and Duration Data, chap. 7, pp. 300-351, Handbook of Applied Econometrics. M.H. Pesaran, ed. (Basil Blackwell), Oxford.

Nickell, S. (1979): "Estimating the Probability of Leaving Unemployment," Econometrica, 47(5), 1249-1266.

OAKes, D. (2001): "Biometrika Centenary: Survival Analysis," Biometrika, 88, 99-142.

Orbe, J., E. Ferreira, And V. Nunez-Anton (2002): "Length of Time Spent in Chapter 11 Bankruptcy: A Censored Partial Regression Model," Applied Economics, 34, 1949-1957.

Richard, J.-F. (1998): "Efficient High-dimensional Monte Carlo Importance Sampling," Working Paper, University of Pittsburgh.

Rosenblatt, M. (1952): "Remarks on a Multivariate Transformation," Annals of Mathematical Statistics, 23, 470-472.

Russell, J. R. (1999): "Econometric Modeling of Multivariate Irregularly-Spaced High-Frequency Data," Working Paper, University of Chicago.

Sueyoshi, G. T. (1995): "A Class of Binary Response Models for Grouped Duration Data," Journal of Applied Econometrics, 10, 411-431.

van den Berg, G. J., AND B. van der KlaAuw (2001): "Combining Micro and Macro Unemployment Duration Data," Journal of Econometrics, 102, 271-309.

Veredas, D., J. Rodriguez-Poo, And A. Espasa (2002): "On the (Intradaily) Seasonality, Dynamics and Durations Zero of a Financial Point Process," CORE DP 2002/23, Louvain-La-Neuve.

Zhang, M. Y., J. Russell, AND R. S. Tsay (2001): "A Nonlinear Autoregressive Conditional Duration Model with Applications to Financial Transaction Data," Journal of Econometrics, 104, 179-207. 